(c) Institute of Mathematical Statistics, 2015

\title{
Estimating Structural Mean Models with Multiple Instrumental Variables Using the Generalised Method of Moments
}

\author{
Paul S. Clarke, Tom M. Palmer and Frank Windmeijer
}

\begin{abstract}
Instrumental variables analysis using genetic markers as instruments is now a widely used technique in epidemiology and biostatistics. As single markers tend to explain only a small proportion of phenotypic variation, there is increasing interest in using multiple genetic markers to obtain more precise estimates of causal parameters. Structural mean models (SMMs) are semiparametric models that use instrumental variables to identify causal parameters. Recently, interest has started to focus on using these models with multiple instruments, particularly for multiplicative and logistic SMMs. In this paper we show how additive, multiplicative and logistic SMMs with multiple orthogonal binary instrumental variables can be estimated efficiently in models with no further (continuous) covariates, using the generalised method of moments (GMM) estimator. We discuss how the Hansen $J$-test can be used to test for model misspecification, and how standard GMM software routines can be used to fit SMMs. We further show that multiplicative SMMs, like the additive SMM, identify a weighted average of local causal effects if selection is monotonic. We use these methods to reanalyse a study of the relationship between adiposity and hypertension using SMMs with two genetic markers as instruments for adiposity. We find strong effects of adiposity on hypertension.
\end{abstract}

Key words and phrases: Structural mean models, multiple instrumental variables, generalised method of moments, Mendelian randomisation, local average treatment effects.

\section{INTRODUCTION}

Additive and multiplicative structural mean models (SMMs) and G-estimation were introduced by Robins $(1989,1994)$ for estimating the causal effects of treat-

Paul S. Clarke is Professor, Institute for Social and Economic Research, University of Essex, Wivenhoe Park, Colchester CO4 3SQ,UK (e-mail: pclarke@essex.ac.uk). Tom M. Palmer is Assistant Professor, Division of Health Sciences, Warwick Medical School, University of Warwick, Coventry CV4 7AL, UK (e-mail:

T.M.Palmer@warwick.ac.uk). Frank Windmeijer is Professor, Centre for Market and Public Organisation and Department of Economics, University of Bristol, 8 Woodland Road, Bristol BS8 1TN, UK (e-mail: f.windmeijer@bristol.ac.uk). ment regimes on outcomes from encouragement designs, namely, randomised controlled trials (RCTs) affected by noncompliance. Additive SMMs are parameterised in terms of average treatment effects and multiplicative SMMs in terms of causal risk ratios; the G-estimators for these models are consistent, asymptotically normal and can be constructed to be semiparametrically efficient. Vansteelandt and Goetghebeur (2003) subsequently developed a class of estimators for generalised SMMs and, in particular, the "double-logistic" SMM for estimating causal odds ratios. Within this literature, causal effects among the treated are identified by the assumption of no effect modification by the instrumental variable (NEM), that is, the causal effect among the treated is the same at each level of the instrumental variable; see, for exam- 
ple, Hernán and Robins (2006). Alternative estimators and identifying assumptions for generalised SMMs have also been developed by Robins and Rotnitzky (2004), Tan (2010) and, for a closely related class of models, van der Laan, Hubbard and Jewell (2007).

The application of SMMs is not limited to encouragement designs, however, and extends to the analysis of observational studies using instrumental variables; see, for example, Hernán and Robins (2006). Instrumental variables analysis involves estimating the causal effect of a temporally antecedent predictor variable on an outcome using an instrumental variable that is associated with the outcome only through its association with the predictor. Instrumental variables analysis has historically been a domain of econometrics, but is now frequently used within epidemiology and biostatistics. In particular, genetic markers were proposed as instruments for modifiable risk factors by Katan (1986) and Davey Smith and Ebrahim (2003). Epidemiological studies using genetic markers are known as Mendelian randomisation studies after the assumption that each individual's genotype is randomly assigned at conception, which implies that the genetic marker is an instrumental variable if it at least partly explains variation in the risk factor. In practice, genetic markers explain only a small proportion of phenotypic variation, and so large sample sizes are required to obtain any reasonable precision. The number of genomewide association studies has increased as the costs of genotyping have decreased, which has led to the identification of multiple genetic variants for the same risk factor. An important attraction of using multiple genetic variants as instrumental variables is that, potentially, more precise causal estimates can be obtained.

Techniques for multiple instruments in linear instrumental variables analysis are already in use; see, for example, Palmer et al. (2012). For linear and nonlinear SMMs, the different frameworks we have mentioned are all general enough to incorporate multiple instrumental variables, but to date the focus in applications has mainly been on cases involving a single instrumental variable. The exceptions are Bowden and Vansteelandt (2011) and Tan (2010). In the first paper, within the frameworks introduced by Robins (1994) and Vansteelandt and Goetghebeur (2003), the authors propose a combination of multiple instrumental variables into a single instrumental variable which, they argue, leads to an optimally efficient estimator. In the second paper, multiple instrumental variables are directly incorporated into the estimating equations, within an alternative framework that introduces new structural models together with doubly robust estimating equations.

In this paper, we consider an alternative framework based on the generalized method of moments (GMM); see, for example, Hansen (1982) and Newey (1993). GMM is widely used in econometrics for the estimation of instrumental variables models. We show how nonlinear SMMs with multiple instruments can be formulated as instrumental variables models and estimated using GMM. Furthermore, if the instrumental variables result in an over-identified model, then the Hansen $J$-test can be used to test parametric identifying assumptions like NEM. We also argue that GMM has good efficiency properties for SMMs without baseline covariates. Specifically, GMM is shown to be semiparametrically efficient in cases where the instrumental variables can be represented by a set of orthogonal binary variables, in which case the efficient combination of the instrumental variables is equivalent to that proposed by Bowden and Vansteelandt (2011). An important practical advantage of GMM is that it can be implemented using existing routines in software packages like Stata and $R$; see Chaussé (2010).

The focus of our presentation is on SMMs without covariates because these models are widely applicable to Mendelian randomisation studies. A drawback to fitting SMMs with covariates using our approach is that the user must correctly specify the covariate effects in a model for the counterfactual exposure-free outcomes, which cannot be tested for misspecification. However, if the covariate effects are saturated-in the sense that the covariates define population strata and the SMM has a separate parameter for the causal effect in each stratum-then this counterfactual model is nonparametric and cannot be misspecified, and the efficiency properties listed above all hold. Saturated SMMs like this can be used to deal with population stratification in Mendelian randomisation studies; see, for example, Lawlor et al. (2008). Tan (2010) also uses GMM but applies it to a very different family of doubly robust estimating equations for which the user must specify the covariate effects in two sets of models; the advantage of this approach is that each model can be tested for misspecification, and the estimator remains consistent for the SMM parameters even if one set of models is misspecified.

In the second part of the paper, we consider the interpretation of additive and multiplicative SMMs with multiple instruments when the key NEM assumption fails. In such circumstances, an additive SMM with 
one binary instrument identifies a "local" average treatment effect (LATE) - also known as a "complier" average causal effect (CACE) - provided that selection is monotonic, and multiplicative SMMs identify local causal risk ratios; see, for example, Clarke and Windmeijer (2010). When there are multiple instruments, Imbens and Angrist (1994) show that a GMM estimator for the additive SMM identifies a weighted average of LATEs. We extend their analysis to multiplicative SMMs to show that a GMM estimator identifies weighted averages of local risk ratios.

To demonstrate our findings, we reanalyse data from a study of the relationship between hypertension and adiposity by Timpson et al. (2009). In the original study, two genetic markers were used as instruments for adiposity and analysed using linear instrumental variables models. We reanalyse this study by focusing on hypertension as a binary outcome and by estimating causal effects of adiposity using multiplicative and logistic SMMs.

The remainder of the paper is organised as follows. In Section 2 we review the potential outcomes framework and the additive, multiplicative and logistic SMMs, first for the simple case of a single binary instrumental variable and then more generally. In Section 3 we show how SMMs with a single binary instrument can be formulated as an instrumental variables model and estimated using GMM, and in Section 4 extend this to multiple instrumental variables. In Section 5 we discuss how GMM combines multiple instruments efficiently for orthogonal binary instruments. In Section 6 we present the results of a Monte Carlo study for multiplicative and logistic SMMs. In Section 7 we derive the multiple instruments results for the local risk ratio. Finally, in Section 8 we apply our estimation procedures to reanalyse the adiposity and hypertension data of Timpson et al. (2009), and in Section 9 make concluding remarks. In the Appendix we provide Stata and $\mathrm{R}$ code for the estimation of the three SMMs using GMM.

\section{STRUCTURAL MEAN MODELS}

\subsection{The Basic Setup}

To introduce SMMs, we follow the exposition in Hernán and Robins (2006) and focus on SMMs for a randomised controlled trial where $Z_{i}, X_{i}$ and $Y_{i}$ are i.i.d. dichotomous random variables for individual subjects $i=1, \ldots, n$ drawn from the target population. For individual $i$, let $Z_{i}$ be a binary indicator of treatment assignment following randomization, $X_{i}$ the selected treatment, and $Y_{i}$ the study outcome. For notational simplicity the subject index is sometimes suppressed for the random variables.

The potential outcomes can now be defined in the usual way. The potential treatments $X_{0}$ and $X_{1}$ are the treatments selected by the individual following assignment to treatment $z=0,1$, respectively. Similarly, the potential (study) outcome $Y_{x z}$ is that obtained if the individual is assigned to treatment $z$ but given treatment $x$. Using potential outcomes notation, we can now state five key conditions that must be satisfied for causal inference: (i) the "stable unit treatment value assumption" that each individual's potential treatments and potential study outcomes are mutually independent of those for any other individual; (ii) the "consistency assumption" $X=X_{Z}$ and $Y=Y_{X Z}$ that links the observed realisations to the potential outcomes; (iii) the "independence assumption", potential outcomes $Y_{z x}$ are independent of $Z$; (iv) the "exclusion restriction" $Y_{x z}=Y_{x}$; and (v) "association assumption", there is an association between $X$ and $Z$. Alternative statements of the key conditions can be found in Robins and Rotnitzky (2004) and Tan (2010).

\subsection{SMM Identification}

For the basic setup defined above, the generalized SMM of Vansteelandt and Goetghebeur (2003) is

$$
\begin{aligned}
h\{ & E(Y \mid X, Z)\}-h\left\{E\left(Y_{0} \mid X, Z\right)\right\} \\
& =\left(\psi_{0}+\psi_{1} Z\right) X,
\end{aligned}
$$

where $Y_{0}$ is often referred to as the exposure-free potential outcome, and $h$ is the link function that determines the interpretation of the target causal parameters $\psi_{0}$ and $\psi_{0}+\psi_{1}$. For example, the identity link leads to the additive SMM $E(Y \mid X, Z)-E\left(Y_{0} \mid X, Z\right)=$ $\left(\psi_{0}+\psi_{1} Z\right) X$, where $\psi_{0}=E\left(Y_{1}-Y_{0} \mid X=1, Z=0\right)$ and $\psi_{0}+\psi_{1}=E\left(Y_{1}-Y_{0} \mid X=Z=1\right)$ are both average treatment effects; the log link leads to the multiplicative $\mathrm{SMM} E(Y \mid X, Z) / E\left(Y_{0} \mid X, Z\right)=\exp \left\{\left(\psi_{0}+\right.\right.$ $\left.\psi_{1} Z\right) X$, where $\exp \left(\psi_{0}\right)=E\left(Y_{1} \mid X=1, Z=0\right) /$ $E\left(Y_{0} \mid X=1, Z=0\right)$ and $\exp \left(\psi_{0}+\psi_{1}\right)=E\left(Y_{1} \mid X=\right.$ $Z=1) / E\left(Y_{0} \mid X=Z=1\right)$ are causal risk ratios.

The SMM parameters are identified by exploiting the conditional mean independence (CMI), or randomisation, assumption

$$
E\left(Y_{0} \mid Z\right)=E\left(Y_{0}\right),
$$

which follows automatically from the key conditions on $Z$ specified above. For the additive SMM, $h$ is the identity link and $E\left(Y_{0} \mid Z\right)=E\left\{Y-\left(\psi_{0}+\right.\right.$ 
$\left.\left.\psi_{1} Z\right) X \mid Z\right\}$; and for the multiplicative SMM, $h=\log$ and $E\left(Y_{0} \mid Z\right)=E\left[Y \exp \left\{-\left(\psi_{0}+\psi_{1} Z\right) X\right\} \mid Z\right]$. However, the CMI assumption (2) alone does not identify $\psi_{0}$ and $\psi_{1}$; for instance, in this simple setup, CMI implies the single independent moment condition

$$
\begin{gathered}
E\left\{Y-\left(\psi_{0}+\psi_{1}\right) X \mid Z=1\right\} \\
=E\left(Y-\psi_{0} X \mid Z=0\right),
\end{gathered}
$$

under the additive SMM. In other words, there is one moment condition with two unknowns. Hence, we must impose dimension-reducing constraints on the SMM. Hernán and Robins (2006) highlight the importance of no effect modification by $Z$ (NEM), which constrains $\psi_{1}=0$ in (3) and identifies $\psi_{0}$. Under NEM, the parameter $\psi_{0}$ of the additive SMM can be interpreted as $E\left(Y_{1}-Y_{0} \mid X=1\right)$, that is, the average causal effect among the treated; and the parameter $\exp \left(\psi_{0}\right)$ of the multiplicative SMM can be interpreted as $E\left(Y_{1} \mid X=1\right) / E\left(Y_{0} \mid X=1\right)$, that is, the causal risk ratio among the treated.

Generally, the form of $E\left(Y_{0} \mid Z\right)$ is more complex than for the additive and multiplicative SMMs because the inverse link function $h^{-1}$ is not separable. Specifically, for the additive SMM, $h=h^{-1}$ is the additively separable identity function [i.e., $h^{-1}(a+b)=$ $\left.h^{-1}(a)+h^{-1}(b)\right]$; and for the multiplicative SMM, $h=\log$ so that $h^{-1}=\exp$ is multiplicatively separable [i.e., $h^{-1}(a+b)=h^{-1}(a) \times h^{-1}(b)$ ]. For nonseparable $h^{-1}$, however, CMI and NEM do not alone identify the parameters of SMMs. For example, the logistic SMM

$$
\begin{aligned}
& \operatorname{logit}\{E(Y \mid X, Z)\}-\operatorname{logit}\left\{E\left(Y_{0} \mid X, Z\right)\right\} \\
& \quad=\left(\psi_{0}+\psi_{1} Z\right) X,
\end{aligned}
$$

where $\operatorname{logit}(p)=\log \{p /(1-p)\}$ and the parameters $\exp \left(\psi_{0}\right)$ and $\exp \left(\psi_{0}+\psi_{1}\right)$ are causal odds ratios for the $(X, Z)=(1,0)$ and $(1,1)$ groups, respectively; assuming that CMI and NEM hold,

$$
\begin{array}{r}
E\left(Y_{0} \mid Z\right)=E[\operatorname{expit}\{\operatorname{logit}(E(Y \mid X, Z)) \\
\left.\left.-\psi_{0} X\right\} \mid Z\right],
\end{array}
$$

where $\operatorname{expit}(a)=\exp (a) /\{1+\exp (a)\}$ is the nonseparable inverse logit function. It is clear that $\psi_{0}$ is not identified unless $E(Y \mid X, Z)$ is known; see, for example, Robins (2000). Hence, to identify $\psi_{0}$, it is necessary to specify an association model

$$
h_{a}\{E(Y \mid X, Z)\}=m_{\beta}(X, Z),
$$

where $h_{a}$ is its link function and $m_{\beta}(X, Z)$ its linear predictor. Vansteelandt and Goetghebeur (2003) specify the double-logistic SMM such that $h_{a}=h=\operatorname{logit}$, where the SMM parameters are identified by the conditional moment conditions

$$
\begin{aligned}
& E\left[\operatorname{expit}\left\{m_{\beta}(X, Z)-\psi_{0} X\right\} \mid Z=0\right] \\
& \quad=E\left[\operatorname{expit}\left\{m_{\beta}(X, Z)-\psi_{0} X\right\} \mid Z=1\right], \\
& E\left[Y-\operatorname{expit}\left\{m_{\beta}(X, Z)\right\} \mid X, Z\right]=0,
\end{aligned}
$$

provided that the association model is correctly specified. A saturated association model is $m_{\beta}(X, Z)=$ $\beta_{0}+\beta_{1} X+\beta_{2} Z+\beta_{3} X Z$ for the simple setup considered here, and is nonparametric in the sense of placing no constraints on the distribution of the observed data. However, nonsaturated logistic association models are potentially uncongenial to the logistic SMM and hence misspecified; see Robins and Rotnitzky (2004). Robins and Rotnitzky (2004) propose an estimator that solves this problem, but Vansteelandt et al. (2011) argue that the impact of an uncongenial association model will be small in practice.

As highlighted by Vansteelandt and Goetghebeur (2005) and Tan (2010), for more general scenarios where any or all of $X, Z$ and $Y$ are nonbinary, NEM is not the only identifying assumption for SMMs. For example, if $Z$ has three categories and $X$ is binary, then CMI implies 3 independent moment conditions, and so the model can be identified if it is correct to assume that $Z$ has a linear effect and the SMM is $\left(\psi_{0}+\psi_{1} Z\right) X$, which identifies both SMM parameters without needing to assume NEM.

\subsection{Estimating Equations}

The construction of consistent estimating equations requires the specification of suitable unconditional moment conditions based on the conditional moment conditions introduced above. The estimating equations are sample analogues of these unconditional moment conditions, and the different estimating approaches in the SMM literature differ in how these unconditional moment conditions are specified. We first consider estimating equations for simple scenarios involving only binary variables, before moving on to the more general case.

Robins (1994) derived G-estimation for additive and multiplicative SMMs. The G-estimator is based on an unconditional moment condition of the form

$$
E\left[\{Z-E(Z)\} E\left(Y_{0} \mid Z\right)\right]=0,
$$

which holds under (2). As shown above, for SMMs with separable inverse link functions, we can write 
$E\left(Y_{0} \mid Z\right)=E\left\{h^{*}\left(X, Y ; \psi_{0}\right) \mid Z\right\}$, where $h^{*}$ is determined by the SMM and NEM is taken to hold. Thus, the sample analogue of (7) is

$$
n^{-1} \sum_{i=1}^{n}\left\{Z_{i}-E(Z)\right\} h^{*}\left(X_{i}, Y_{i} ; \widehat{\psi}_{0}\right)=0,
$$

where, for example, $h^{*}\left(X, Y ; \widehat{\psi}_{0}\right)=Y-\widehat{\psi}_{0} X$ for the additive $\mathrm{SMM}$ and $h^{*}\left(X, Y ; \widehat{\psi}_{0}\right)=Y \exp \left(-\widehat{\psi}_{0} X\right)$ under the multiplicative SMM. Under regularity conditions, $\widehat{\psi}_{0}$ is a consistent estimator for $\psi_{0}$ under CMI provided that (a) the SMM is correctly specified and (b) $E(Z)$ is known. The second of these conditions will be satisfied if $Z$ is based on a known allocation rule such as randomisation. Otherwise, if $E(Z)$ is unknown, we must specify a (trivial) model $E(Z)=\mu$ and replace $E(Z)$ in (8) with $\widehat{\mu}$, that is, a consistent estimator of $\mu$. Robins, Mark and Newey (1992) note that the correct asymptotic covariance matrix for $\widehat{\psi}_{0}$ can only be derived from an extended system of moment conditions that includes $E(Z-\mu)=0$; see also Vansteelandt and Goetghebeur (2003) and Tan (2010). Conversely, treating $\widehat{\mu}$ as known when deriving the asymptotic variance of $\widehat{\psi}_{0}$ leads to an expression that is too large, and results in conservative inferences; see Robins, Mark and Newey (1992) and Vansteelandt and Goetghebeur (2003).

The estimating equations for the double-logistic SMM are

$$
n^{-1} \sum_{i=1}^{n}\left(Z_{i}-\mu\right) \operatorname{expit}\left\{m_{\boldsymbol{\beta}}(X, Z)-\psi_{0} X\right\}=0
$$

where $\mu=E(Z)$ as before. Due to the nonseparability of the expit function, the estimating equation involves the association model $\operatorname{logit}\{E(Y \mid X, Z)\}=m_{\beta}(X, Z)$. As with $\mu$, we must replace $\boldsymbol{\beta}$ in (9) with a consistent estimator $\widehat{\boldsymbol{\beta}}$, and the correct asymptotic covariance can only be derived from a set of moment conditions that includes ones for $m_{\beta}(X, Z)$ as well as for $\mu$. Conservative inferences again result if $\widehat{\boldsymbol{\beta}}$ is treated as known when deriving the asymptotic covariance matrix.

More generally, for models involving multiple or continuous instrumental variables, the estimators above are based on unconditional moment conditions of the form

$$
E\left[\left\{d(Z)-\mu_{d}\right\} E\left(Y_{0} \mid Z\right)\right]=0,
$$

where $E\left(Y_{0} \mid Z\right)$ is determined by the SMM, $d(Z)$ is a user-specified function, and $\mu_{d}=E\{d(Z)\}$. The choice of $d(Z)$ does not affect consistency but does affect efficiency. Robins (1994) derives the choice of
$d(Z)=d_{\mathrm{opt}}(Z)$ for the additive and multiplicative SMMs so that the first-order asymptotitc variance is minimised and the estimator is semiparametrically efficient; Vansteelandt and Goetghebeur (2003) derive the equivalent choice for the double-logistic SMM. For further details see, for example, Tsiatis (2006) and Bowden and Vansteelandt (2011).

\subsection{Covariates}

In this paper we focus mainly on SMMs that do not condition on baseline covariates, but for completeness we discuss here the estimation of SMMs which do include covariates; the treatment of covariates is discussed further in Section 9. A generalised SMM with baseline covariates $\mathbf{C}$ has the form

$$
\begin{aligned}
& h\{E(Y \mid X, Z, \mathbf{C})\}-h\left\{E\left(Y_{0} \mid X, Z, \mathbf{C}\right)\right\} \\
& \quad=\eta_{\psi}(X, Z, \mathbf{C}),
\end{aligned}
$$

where $\psi$ is the SMM parameter vector and $\eta_{\psi}(X, Z$, C) must satisfy $\eta_{\psi}(0, Z, \mathbf{C})=0$. If $h^{-1}$ is nonseparable, then the association model is specified as $h\{E(Y \mid X, Z, \mathbf{C})\}=m_{\boldsymbol{\beta}}(X, Z, \mathbf{C})$. In terms of identifying assumptions, $\mathrm{CMI}$ is now conditional on baseline C such that

$$
E\left(Y_{0} \mid Z, \mathbf{C}\right)=E\left(Y_{0} \mid \mathbf{C}\right),
$$

where NEM corresponds to $\eta_{\psi}(X, Z, \mathbf{C})=\eta_{\psi}(X, \mathbf{C})$ and alternative dimension-reducing parametric constraints are discussed by Vansteelandt and Goetghebeur (2005) and Tan (2010). Finally, the unconditional moment condition (10) on which the estimating equations are based becomes

$$
E\left[\left\{d(Z, \mathbf{C})-\mu_{d}(\mathbf{C})\right\} E\left(Y_{0} \mid Z, \mathbf{C}\right)\right]=0,
$$

where $E\left(Y_{0} \mid Z, \mathbf{C}\right)$ is determined, as before, by the SMM, $E\left(Y_{0} \mid Z, \mathbf{C}\right)=d(Z, \mathbf{C})$ is a user-specified function, and $\mu_{d}(\mathbf{C})=E\{d(Z, \mathbf{C}) \mid \mathbf{C}\}$. Consistency thus depends on correctly specifying the conditional distribution of $Z$ given $\mathbf{C}$ so that $\mu_{d}(\mathbf{C})$ is correct for given $d$. Robins (1994) and Vansteelandt and Goetghebeur (2003) derive the optimal choices of $d$ for additive, multiplicative and double-logistic SMMs when $\operatorname{Pr}(Z=z \mid \mathbf{C})$ is presumed to be known; see also Bowden and Vansteelandt (2011).

An important special case for Mendelian randomisation studies is where there are discrete baseline covariates to handle population stratification; see, for example, Lawlor et al. (2008). The generalized SMM with saturated covariate effects can be written

$$
\begin{aligned}
& h\{E(Y \mid X, Z, \mathbf{C}=\mathbf{c})\}-h\left\{E\left(Y_{0} \mid X, Z, \mathbf{C}=\mathbf{c}\right)\right\} \\
& \quad=X \psi_{\mathbf{c}},
\end{aligned}
$$


where NEM is taken to hold, and $\psi_{\mathbf{c}}$ is a unique parameter for the population in the stratum defined by $\mathbf{C}=\mathbf{c}$. Saturated models of this form are equivalent to specifying separate no-covariate SMMs within each stratum. Therefore, it can be shown that all of the results in this paper regarding no-covariate SMMs also apply to saturated-covariate SMMs; see also Angrist and Imbens (1995), Theorem 3.

Tan (2010) develops an alternative family of doubly robust estimating equations specifically for generalised SMMs with nonseparable inverse link functions that include continuous covariates. Furthermore, he allows for the inclusion of an extended set of covariates $\mathbf{V}$ that includes $\mathbf{C}$ so that additional covariates predictive of $Z$, $X$ and $Y$ can be incorporated. The analyst first chooses a working distribution $p^{*}(z \mid \mathbf{c})$ for $\operatorname{Pr}(Z=z \mid \mathbf{C}=\mathbf{c})$ that is arbitrary and so does not have to be correct. The analyst must then specify two sets of parametric models involving the full covariates $\mathbf{V}$ : (a) $\operatorname{Pr}(Z=$ $z \mid \mathbf{V}=\mathbf{v})=k_{\lambda}(z \mid \mathbf{v})$; and (b) $\operatorname{Pr}(X=x \mid Z=z, \mathbf{V}=$ $\mathbf{v})=g_{\alpha}(x \mid z, \mathbf{v})$ and $E(Y \mid X, Z, \mathbf{V})=m_{v}^{*}(X, Z, \mathbf{V})$. Using the law of iterated expectations, it can be shown that the following estimating equation is consistent for $\widehat{\psi}$ if either model (a) or model (b) are misspecified (but not both):

$$
\begin{aligned}
n^{-1} \sum_{i}\left[\frac{p^{*}\left(Z_{i} \mid \mathbf{C}_{i}\right)}{k_{\hat{\lambda}}\left(Z_{i} \mid \mathbf{V}_{i}\right)} \phi^{i} \Delta_{\widehat{\psi}, \widehat{\boldsymbol{\beta}}}^{i}\right. \\
\left.\quad-\left\{\frac{p^{*}\left(Z_{i} \mid \mathbf{C}_{i}\right)}{k_{\widehat{\lambda}}\left(Z_{i} \mid \mathbf{V}_{i}\right)} \phi^{i} \widehat{w}^{i}-E_{Z}^{*}\left(\phi^{i} \widehat{w}^{i}\right)\right\}\right]=0,
\end{aligned}
$$

where $\Delta_{\boldsymbol{\psi}, \boldsymbol{\beta}}^{i}=Y_{i}-h^{-1}\left\{m_{\boldsymbol{\beta}}\left(X_{i}, Z_{i}, \mathbf{C}_{i}\right)\right\}+$ $h^{-1}\left\{m_{\boldsymbol{\beta}}\left(X_{i}, Z_{i}, \mathbf{C}_{i}\right)-\eta_{\psi}\left(X_{i}, Z_{i}, \mathbf{C}_{i}\right)\right\}$,

$$
\begin{array}{r}
\widehat{w}^{i}=\sum_{x^{\prime}} g_{\widehat{\alpha}}\left(x^{\prime} \mid Z_{i}, \mathbf{V}_{i}\right)\left[h^{-1}\left\{m_{\widehat{\boldsymbol{v}}}^{*}\left(x^{\prime}, Z_{i}, \mathbf{V}_{i}\right)\right\}\right. \\
\left.+\Delta_{\widehat{\boldsymbol{\psi}}, \widehat{\boldsymbol{\beta}}}^{i}-Y_{i}\right],
\end{array}
$$

is an estimator of $E\left(\Delta_{\psi, \beta}^{i} \mid Z, \mathbf{V}\right)$, and $E_{Z \mid \mathbf{C}=\mathbf{c}}^{*}(\cdot)=$ $\sum_{z^{\prime}} p^{*}\left(z^{\prime} \mid \mathbf{c}\right)(\cdot)$ if $Z$ is discrete. Three important features to note are that $\Delta_{\widehat{\boldsymbol{\psi}}, \widehat{\boldsymbol{\beta}}}^{i}-Y_{i}$ does not depend on $Y_{i}, \Delta_{\boldsymbol{\psi}, \boldsymbol{\beta}}^{i}$ is the key to identification because $E\left\{E\left(\Delta_{\psi, \beta}^{i} \mid Z_{i}, \mathbf{V}_{i}\right) \mid \mathbf{C}_{i}\right\}=E\left(Y_{i 0} \mid Z_{i}, \mathbf{C}_{i}\right)$, and, while $p^{*}$ does not need to be correctly specified, one must construct $\phi^{i}=d\left(Z_{i}, \mathbf{C}_{i}\right)-\mu^{*}\left(\mathbf{C}_{i}\right)$ for user-specified $d$ where $\mu^{*}(\mathbf{C})=E_{Z \mid \mathbf{C}}^{*}\{d(Z, \mathbf{C})\}$. Tan (2010) also considers other doubly robust estimating schemes and argues that the estimator based on the estimating equations above is locally efficient given the analyst's choices of $p^{*}$ and $d$.

\section{THE GENERALISED METHOD OF MOMENTS}

In this section we propose an alternative approach to constructing estimating equations based on the generalized method of moments (GMM). Hansen (1982) proposed GMM for moment-condition models of the form $E(\mathbf{g}(\boldsymbol{\delta}))=\mathbf{0}$, where $\mathbf{g}(\boldsymbol{\delta})$ is a random vector and a function of parameter $\boldsymbol{\delta}$, and $\mathbf{0}$ is an appropriately dimensioned column vector of zeros. A general expression for the GMM estimator is given by

(11) $\widehat{\boldsymbol{\delta}}=\underset{\boldsymbol{\delta}}{\arg \min }\left\{n^{-1} \sum_{i=1}^{n} \mathbf{g}_{i}^{\prime}(\boldsymbol{\delta})\right\} W_{n}^{-1}\left\{n^{-1} \sum_{i=1}^{n} \mathbf{g}_{i}(\boldsymbol{\delta})\right\}$,

where $\mathbf{g}_{i}(\boldsymbol{\delta})$ is the random vector for subject $i, \mathbf{g}_{i}^{\prime}(\boldsymbol{\delta})$ is its transpose, and $W_{n}$ is a user-chosen weight-matrix that determines the efficiency of the estimator. Tan (2010) has applied the theory of GMM to the doubly robust estimating equations discussed in the previous section, but the focus here is on its use in econometrics for instrumental variables models of the form

$$
\mathbf{g}(\boldsymbol{\delta})=v(\boldsymbol{\delta}) \mathbf{S},
$$

where $v(\boldsymbol{\delta})$ is known as the generalized residual and $\mathbf{S}$ is a random vector of instrumental variables. The generalized residual is so called because it satisfies $E(v(\boldsymbol{\delta}) \mid \mathbf{S})=0$. We show how any nonlinear SMM can be expressed as an instrumental variables model by exploiting that $E\left\{Y_{0}-E\left(Y_{0}\right) \mid Z\right\}=0$ under CMI (2) and by developing estimating equations which are sample analogues of

$$
E\left[d(\mathbf{S}) E\left\{Y_{0}-E\left(Y_{0}\right) \mid \mathbf{S}\right\}\right]=0,
$$

where $d(\mathbf{S})$ is a user-specified function that affects efficiency but not consistency. The choice of $d(\mathbf{S})$ that minimises the variance of the GMM estimator, the socalled efficient instrument, depends on $W_{n}$ and will be discussed further on.

In our simple scenario involving only binary variables, the SMM is just identified in the sense that it has one parameter and one moment condition under CMI (for now taking $\beta$ to be known for the double-logistic SMM). For example, the additive SMM under NEM leads to the well-known estimator

$$
\widehat{\psi}_{0}=\frac{E(Y \mid Z=1)-E(Y \mid Z=0)}{E(X \mid Z=1)-E(X \mid Z=0)},
$$

in this case, namely, the classical instrumental variable estimator; see, for example, Hernán and Robins (2006). Theory based on the GMM estimator (11) is not needed here because $\widehat{\psi}_{0}$ is simply the solution to (3) under NEM, and the choice of $d(\mathbf{S})$ is irrelevant because $Z$ 
is binary. However, we can use this simple example to show how the additive SMM can be specified as an instrumental variables model.

First, the CMI moment condition can be written as $E\left(Y_{0} \mid Z=z\right)-\alpha_{0}=0$ for $z=0,1$, where $E\left(Y_{0}\right)$ is simply treated as an extra parameter $\alpha_{0}$ and results in the additional moment condition $E\left(Y_{0}\right)-\alpha_{0}=0$. It follows that one of $E\left(Y_{0} \mid Z=z\right)-\alpha_{0}=0$ is redundant because $Z$ is discrete and $E\left\{E\left(Y_{0} \mid Z\right)\right\}=\alpha_{0}$ by definition. However, using the additional $E\left(Y_{0}\right)-\alpha_{0}=0$ moment condition allows the system of moment conditions to be expressed in terms of a generalised residual and a vector of instrumental variables as in (12). For example, under the additive SMM, it follows that

$$
\begin{aligned}
& {\left[\begin{array}{c}
E\left(Y-\psi_{0} X\right)-\alpha_{0} \\
E\left(Y-\psi_{0} X \mid Z=1\right)-\alpha_{0}
\end{array}\right]=\left(\begin{array}{l}
0 \\
0
\end{array}\right)} \\
& \Rightarrow E\left[\begin{array}{c}
Y-\psi_{0} X-\alpha_{0} \\
\left(Y-\psi_{0} X-\alpha_{0}\right) Z
\end{array}\right]=\left(\begin{array}{l}
0 \\
0
\end{array}\right),
\end{aligned}
$$

that is, $E\left\{\mathbf{g}\left(\psi_{0}, \alpha_{0}\right)\right\}=\mathbf{0}$, where $\mathbf{g}\left(\psi_{0}, \alpha_{0}\right)=(Y-$ $\left.\psi_{0} X-\alpha_{0}\right) \mathbf{S}$ and $\mathbf{S}=(1, Z)^{\prime}$. Similarly, for the multiplicative SMM, it follows that

$$
E\left[\begin{array}{c}
Y \exp \left(-\psi_{0} X\right)-\alpha_{0} \\
\left\{Y \exp \left(-\psi_{0} X\right)-\alpha_{0}\right\} Z
\end{array}\right]=\left(\begin{array}{l}
0 \\
0
\end{array}\right)
$$

and for the double-logistic SMM with a saturated association model,

$$
\begin{aligned}
& E\left[\begin{array}{c}
\operatorname{expit}\left(\beta_{0}+\beta_{1} X+\beta_{2} Z\right. \\
\left.+\beta_{3} X Z-\psi_{0} X\right)-\alpha_{0} \\
\left\{\operatorname { e x p i t } \left(\beta_{0}+\beta_{1} X+\beta_{2} Z\right.\right. \\
\left.\left.+\beta_{3} X Z-\psi_{0} X\right)-\alpha_{0}\right\} Z
\end{array}\right] \\
& \quad=\left(\begin{array}{l}
0 \\
0
\end{array}\right) .
\end{aligned}
$$

The estimators for these three models are trivial special cases of GMM because each is just identified, but it is clear that moment conditions (14)-(15) are of the form $E(v(\boldsymbol{\delta}) \mathbf{S})=\mathbf{0}$, where $\mathbf{0}$ is an appropriately dimensioned vector of zeros. It is also clear that moment condition (16) for the double-logistic SMM has the more complicated form $E\{\mathbf{g}(\boldsymbol{\delta} ; \boldsymbol{\beta})\}=\mathbf{0}$, because the vector of association model parameters $\boldsymbol{\beta}$ is usually unknown. We now discuss what happens when $\mathbf{S}$ is expanded to include multiple instrumental variables.

\section{MULTIPLE INSTRUMENTS}

Mendelian randomisation studies justify the use of genetic markers as instrumental variables by arguing that (a) the random allocation of genes from parents to offspring mimics a randomised experiment, and (b) there is an established relationship between the marker and some modifiable risk factor of interest; see, for example, Katan (1986), Davey Smith and Ebrahim (2003) and Lawlor et al. (2008).

The genetic variant typically has three forms: homozygous for the common allele; heterozygous; and homozygous for the rare allele. If we code these 0,1 and 2, respectively, then the resulting instrument $Z$ is multivalued. In fact, this is a simple multiple instruments example because the three-level variable can be coded using two orthogonal binary variables, for example, $Z_{1}=I(Z=1)$ and $Z_{2}=I(Z=2)$, where $I$ is the indicator function.

\subsection{Additive SMM}

The additive SMM for multiple instruments in this case can be written as

$$
\begin{aligned}
& E\left(Y \mid X, Z_{1}, Z_{2}\right)-E\left(Y_{0} \mid X, Z_{1}, Z_{2}\right) \\
& \quad=\left(\psi_{0}+\psi_{1} Z_{1}+\psi_{2} Z_{2}\right) X,
\end{aligned}
$$

where NEM corresponds to constraining $\psi_{1}=\psi_{2}=0$ and CMI yields the moment conditions

$$
\left\{\begin{array}{c}
E\left(Y-\psi_{0} X-\alpha_{0}\right) \\
E\left(Y-\psi_{0} X-\alpha_{0} \mid Z_{1}=1\right) \\
E\left(Y-\psi_{0} X-\alpha_{0} \mid Z_{2}=1\right)
\end{array}\right\}=\left(\begin{array}{l}
0 \\
0 \\
0
\end{array}\right),
$$

where $\alpha_{0}=E\left(Y_{0}\right)$ as before. The unconditional moment condition is

$$
E\left\{\left(Y-\psi_{0} X-\alpha_{0}\right) \mathbf{S}\right\}=\mathbf{0},
$$

where $\mathbf{S}=\left(1, Z_{1}, Z_{2}\right)^{\prime}$ is a random vector representing the multiple instruments; note that $\mathbf{S}$ is orthogonal because its elements are mutually exclusive such that $\mathbf{S S}^{\prime}=\operatorname{diag}(\mathbf{S})$. In fact, this model is linear and so the parameters can be consistently estimated using standard Two-Stage Least Squares (2SLS). The 2SLS estimator can be obtained as the ordinary least squares (OLS) estimator from regressing $Y$ on $\widehat{X}$, where $\widehat{X}$ is the prediction from the first-stage regression of $X$ on $\mathbf{S}$. The 2SLS estimator is a special case of a "onestep" GMM estimator with $W_{n}=n^{-1} \sum_{i} \mathbf{S}_{i} \mathbf{S}_{i}^{\prime}$ (see next section), and is commonly used for linear instrumental variables analysis with multiple instruments; see Palmer et al. (2012) for its use with Mendelian randomisation studies.

\subsection{Multiplicative SMM}

The saturated multiplicative SMM for the two instruments is

$$
\begin{array}{r}
E\left(Y \mid X, Z_{1}, Z_{2}\right) / E\left(Y_{0} \mid X, Z_{1}, Z_{2}\right) \\
=\exp \left\{\left(\psi_{0}+\psi_{1} Z_{1}+\psi_{2} Z_{2}\right) X\right\}
\end{array}
$$


where NEM here corresponds to $\psi_{1}=\psi_{2}=0$. Using the same vector of instrumental variables $\mathbf{S}$, the multiplicative SMM moment conditions can be written as

$$
E\left[\left\{\frac{Y}{\exp \left(X \psi_{0}\right)}-\alpha_{0}\right\} \mathbf{S}\right]=\mathbf{0} .
$$

Letting $\alpha_{0}^{*}=\log \left(\alpha_{0}\right)$, it is easy to show that (17) also implies

$$
E\left\{\frac{Y-\exp \left(\alpha_{0}^{*}+X \psi_{0}\right)}{\exp \left(X \psi_{0}\right)} \mathbf{S}\right\}=\mathbf{0}
$$

and

$$
E\left\{\frac{Y-\exp \left(\alpha_{0}^{*}+X \psi_{0}\right)}{\exp \left(\alpha_{0}^{*}+X \psi_{0}\right)} \mathbf{S}\right\}=\mathbf{0},
$$

where (19) is obtained simply by dividing (18) by $\exp \left(\alpha_{0}^{*}\right) \neq 0$. Moment condition (19) is the same as that for exponential-mean models proposed by Mullahy (1997).

For example, consider a GMM estimator based on moment condition (17). The GMM estimator for $\boldsymbol{\delta}=\left(\alpha_{0}, \psi_{0}\right)^{\prime}$ is the solution to (11) with $\mathbf{g}(\boldsymbol{\delta})=$ $\left\{Y \exp \left(-X \psi_{0}\right)-\alpha_{0}\right\} \mathbf{S}$. The one-step GMM estimator $\widehat{\boldsymbol{\delta}}_{1}$ is obtained by choosing the weight matrix in (11) to be $W_{n}=n^{-1} \sum_{i} \mathbf{S}_{i} \mathbf{S}_{i}^{\prime}$. The two-step GMM estimator $\widehat{\boldsymbol{\delta}}_{2}$ is obtained by estimating the weight matrix

$$
W_{n}\left(\widehat{\boldsymbol{\delta}}_{1}\right)=n^{-1} \sum_{i=1}^{n} \mathbf{g}_{i}\left(\widehat{\boldsymbol{\delta}}_{1}\right) \mathbf{g}_{i}^{\prime}\left(\widehat{\boldsymbol{\delta}}_{1}\right),
$$

using the one-step GMM estimator $\widehat{\boldsymbol{\delta}}_{1}$. Under standard regularity conditions, the limiting distributions of the one-step and two-step GMM estimators are

$$
\begin{gathered}
n^{1 / 2}\left(\widehat{\boldsymbol{\delta}}_{1}-\boldsymbol{\delta}_{0}\right) \stackrel{d}{\longrightarrow} N\left\{\mathbf{0},\left(C_{0}^{\prime} W^{-1} C_{0}\right)^{-1} C_{0} W^{-1}\right. \\
\left.\cdot \Omega_{0} W^{-1} C_{0}\left(C_{0}^{\prime} W^{-1} C_{0}\right)^{-1}\right\}, \\
n^{1 / 2}\left(\widehat{\boldsymbol{\delta}}_{2}-\boldsymbol{\delta}_{0}\right) \stackrel{d}{\longrightarrow} N\left\{\mathbf{0},\left(C_{0}^{\prime} \Omega_{0}^{-1} C_{0}\right)^{-1}\right\},
\end{gathered}
$$

respectively, where $\boldsymbol{\delta}_{0}$ is the true parameter value, $\stackrel{d}{\longrightarrow}$ indicates convergence in distribution, $N$ indicates a normally distributed random vector,

$$
C_{0}=E\left\{\frac{\partial \mathbf{g}\left(\boldsymbol{\delta}_{0}\right)}{\partial \boldsymbol{\delta}^{\prime}}\right\}, \quad \Omega_{0}=E\left\{\mathbf{g}\left(\boldsymbol{\delta}_{0}\right) \mathbf{g}^{\prime}\left(\boldsymbol{\delta}_{0}\right)\right\},
$$

and $W=E\left(\mathbf{S}_{i} \mathbf{S}_{i}^{\prime}\right)$ is the probability limit of the onestep GMM estimator's weight matrix.

Chamberlain (1987) shows that the two-step GMM estimator is semiparametrically efficient when the instruments are mutually exclusive indicators that follow a multinomial distribution, as is the case in this example provided that there are no continuous covariates or instruments. More generally, as will be discussed in Section 5, one must derive the efficient instrument $d(\mathbf{S})=d_{\text {opt }}(\mathbf{S})$ for the GMM estimator to be semiparametrically efficient.

A useful property of two-step GMM for overidentified models is that it admits the use of the Hansen $J$-test, which can be used to assess the validity of the moment conditions; see Hansen (1982). The test statistic and its limiting distribution (under the null hypothesis that the moment conditions are valid) are given by

$$
\begin{aligned}
J\left(\widehat{\boldsymbol{\delta}}_{2}\right)= & n\left\{n^{-1} \sum_{i=1}^{n} \mathbf{g}_{i}^{\prime}\left(\widehat{\boldsymbol{\delta}}_{2}\right)\right\} \\
& \cdot W_{n}^{-1}\left(\widehat{\boldsymbol{\delta}}_{1}\right)\left\{n^{-1} \sum_{i=1}^{n} \mathbf{g}_{i}\left(\widehat{\boldsymbol{\delta}}_{2}\right)\right\} \\
\stackrel{d}{\longrightarrow} & \chi_{q}^{2},
\end{aligned}
$$

where $\chi_{q}^{2}$ indicates a chi-squared random variable with $q$ degrees of freedom, and $q$ is the number of moment conditions by which the model is over identified (e.g., $q=1$ in this illustration).

\subsection{Double-Logistic SMM}

Under NEM, the logistic SMM for the two instruments is

$$
\begin{aligned}
& \operatorname{logit}\left\{E\left(Y \mid X, Z_{1}, Z_{2}\right)\right\}-\operatorname{logit}\left\{E\left(Y_{0} \mid X, Z_{1}, Z_{2}\right)\right\} \\
& \quad=\psi_{0} X,
\end{aligned}
$$

and its association model is

$$
E\left(Y \mid X, Z_{1}, Z_{2}\right)=\operatorname{expit}\left\{m_{\beta}\left(X, Z_{1}, Z_{2}\right)\right\},
$$

where $m_{\beta}\left(X, Z_{1}, Z_{2}\right)=\beta_{0}+\beta_{1} X+\beta_{2} Z_{1}+\beta_{3} Z_{2}+$ $\beta_{4} X Z_{1}+\beta_{5} X Z_{2}$ is saturated. We describe two estimation methods: first, where the parameters in the saturated association model are estimated by maximum likelihood and then plugged into the estimating equations for the double-logistic SMM; and, second, where all parameters are estimated jointly in a similar manner to that proposed by Vansteelandt and Goetghebeur (2003) and Bowden and Vansteelandt (2011).

Denoting $\widehat{\boldsymbol{\beta}}$ as the maximum likelihood estimator of $\boldsymbol{\beta}$, it follows that

$$
E\{\mathbf{g}(\boldsymbol{\delta} ; \widehat{\boldsymbol{\beta}})\}=E\left[\left\{q\left(\psi_{0} ; \widehat{\boldsymbol{\beta}}\right)-\alpha_{0}\right\} \mathbf{S}\right]=\mathbf{0},
$$

where $\boldsymbol{\delta}=\left(\psi_{0}, \alpha_{0}\right)^{\prime}, q\left(\psi_{0} ; \boldsymbol{\beta}\right)=\operatorname{expit}\left\{m_{\boldsymbol{\beta}}\left(X, Z_{1}\right.\right.$, $\left.\left.Z_{2}\right)-X \psi_{0}\right\}$ and $\mathbf{S}=\left(1, Z_{1}, Z_{2}\right)^{\prime}$. Point estimation is carried out exactly as before, but standard error estimates obtained by fixing $\widehat{\boldsymbol{\beta}}$ and plugging it into the 
asymptotic covariance matrices presented above will be biased because the first stage estimation of $\boldsymbol{\beta}$ is ignored; see the discussion in Section 2.3. However, theory for "two-stage" GMM estimators (2SGMM) has been developed by Gouriéroux, Monfort and Renault (1996). The $2 \mathrm{SGMM} \widehat{\boldsymbol{\delta}}_{1, \boldsymbol{\beta}}$ is the solution to (11) and its asymptotic distribution is

$$
\begin{aligned}
& n^{1 / 2}\left(\widehat{\boldsymbol{\delta}}_{1, \boldsymbol{\beta}}-\boldsymbol{\delta}_{0}\right) \\
& \quad \stackrel{d}{\longrightarrow} N\left\{\mathbf{0},\left(C_{0}^{\prime} W C_{0}\right)^{-1} C_{0} W \Omega_{0}^{*} W C_{0}\left(C_{0}^{\prime} W C_{0}\right)^{-1}\right\},
\end{aligned}
$$

where $C_{0}$ and $W$ are both defined as above, and $\Omega_{0}^{*}$ is the asymptotic variance of the limiting normal distribution of

$$
n^{-1 / 2} \sum_{i=1}^{n} \mathbf{g}_{i}\left(\boldsymbol{\delta}_{0} ; \boldsymbol{\beta}_{0}\right)+E\left\{\frac{\partial \mathbf{g}\left(\boldsymbol{\delta}_{0} ; \boldsymbol{\beta}_{0}\right)}{\partial \boldsymbol{\beta}^{\prime}}\right\} n^{1 / 2}\left(\widehat{\boldsymbol{\beta}}-\boldsymbol{\beta}_{0}\right),
$$

which has the consistent estimator

$$
\begin{aligned}
n \widehat{\Omega}^{*}= & \sum_{i=1}^{n} \widehat{\mathbf{g}}_{i} \widehat{\mathbf{g}}_{i}^{\prime}+\widehat{G}_{\boldsymbol{\beta}}^{\prime} \widehat{V(\widehat{\boldsymbol{\beta}})} \widehat{G}_{\boldsymbol{\beta}} \\
& +\widehat{G}_{\boldsymbol{\beta}}^{\prime} \widehat{V(\widehat{\boldsymbol{\beta}})}\left(\sum_{i=1}^{n} Q_{i} \mathbf{R}_{i} \widehat{\mathbf{g}}_{i}^{\prime}\right) \\
& +\left(\sum_{i=1}^{n} Q_{i} \widehat{\mathbf{g}}_{i} \mathbf{R}_{i}^{\prime}\right) \widehat{V(\widehat{\boldsymbol{\beta}})} \widehat{G}_{\boldsymbol{\beta}},
\end{aligned}
$$

with $\widehat{\mathbf{g}}_{i}=\mathbf{g}_{i}\left(\widehat{\boldsymbol{\delta}}_{1, \boldsymbol{\beta}} ; \widehat{\boldsymbol{\beta}}\right), \quad \widehat{G}_{\boldsymbol{\beta}}=\sum_{i} \partial \mathbf{g}_{i}^{\prime}\left(\widehat{\boldsymbol{\delta}}_{1, \boldsymbol{\beta}} ; \widehat{\boldsymbol{\beta}}\right) / \partial \boldsymbol{\beta}$, $\widehat{V(\widehat{\boldsymbol{\beta}})}=\left(\sum_{i} \widehat{p}_{i}\left(1-\widehat{p}_{i}\right) \mathbf{R}_{i} \mathbf{R}_{i}^{\prime}\right)^{-1}, \mathbf{R}_{i}=\left(1, X_{i}, Z_{1 i}\right.$, $\left.Z_{2 i}, X_{i} Z_{1 i}, X_{i} Z_{2 i}\right)^{\prime}, \widehat{p}_{i}=\operatorname{expit}\left\{m_{\widehat{\boldsymbol{\beta}}}\left(X_{i}, Z_{i 1}, Z_{i 2}\right)\right\}$ and $Q_{i}=Y_{i}-\widehat{p}_{i}$. Furthermore, $\widehat{\Omega}^{*}$ is also the weight matrix for the asymptotically efficient two-step 2SGMM estimator, and so the limiting distribution of the Hansen $J$-test statistic (with $W_{n}=\widehat{\Omega}^{*}$ ) is also valid.

Vansteelandt and Goetghebeur (2003) developed estimating equations for the double-logistic SMM by expanding its system of estimating equations to include those for the association model. As in Bowden and Vansteelandt (2011), a joint GMM estimator can be obtained by applying the GMM estimator to

$$
\begin{aligned}
& \mathbf{g}(\boldsymbol{\delta} ; \boldsymbol{\beta}) \\
& \quad=\left(\begin{array}{c}
{\left[Y-\operatorname{expit}\left\{m_{\boldsymbol{\beta}}\left(X, Z_{1}, Z_{2}\right)\right\}\right] \mathbf{R}} \\
{\left[\operatorname{expit}\left\{m_{\boldsymbol{\beta}}\left(X, Z_{1}, Z_{2}\right)-\psi_{0} X-\alpha_{0}\right\}\right] \mathbf{S}}
\end{array}\right),
\end{aligned}
$$

where $\mathbf{R}$ is defined above and $\boldsymbol{\delta}=\left(\alpha_{0}, \psi_{0}\right)^{\prime}$. Gouriéroux, Monfort and Renault (1996) show that the asymptotic distributions of the 2 SGMM and the joint GMM estimators are the same. An important advantage of using the joint moments (22) is that standard
GMM software can be used to make asymptotically correct inferences about the target parameter $\psi_{0}$. Further details on how the gmm command in Stata and the $g m m($ ) function in $\mathrm{R}$ can be used to implement these estimators are given in the Appendix.

\section{COMBINING MULTIPLE INSTRUMENTS}

Bowden and Vansteelandt (2011) derive the optimally efficient combination of instruments and, for practical purposes, a simplified expression for this combination. We consider the particular case of SMMs without covariates where identification is obtained using orthogonal binary instruments. In such cases, we show that the one-step GMM estimator combines the instruments as in Bowden and Vansteelandt (2011) under the simplifying assumption of a constant variance, and that the two-step GMM estimator combines the instruments optimally.

First consider the one-step GMM estimator by noting that it is the solution to the first derivative of (11) evaluated at zero. For the multiplicative SMM based on (17), this gives

$$
\begin{gathered}
\left\{n^{-1} \sum_{i=1}^{n} \frac{\partial \mathbf{g}_{i}^{\prime}(\boldsymbol{\delta})}{\partial \boldsymbol{\delta}}\right\} W_{n}^{-1}\left\{n^{-1} \sum_{i=1}^{n} \mathbf{g}_{i}(\boldsymbol{\delta})\right\} \\
=\left\{n^{-1} \sum_{i=1}^{n}\left(\begin{array}{c}
1 \\
Y_{i} X_{i} \exp \left(-X_{i} \psi_{0}\right)
\end{array}\right) \mathbf{S}_{i}^{\prime}\right\} \\
\cdot W_{n}^{-1}\left\{n^{-1} \sum_{i=1}^{n} \mathbf{g}_{i}(\boldsymbol{\delta})\right\}=\mathbf{0},
\end{gathered}
$$

where $\mathbf{g}_{i}(\boldsymbol{\delta})=\left\{Y \exp \left(-X \psi_{0}\right)-\alpha_{0}\right\} \mathbf{S}$. This system can be expressed as

$$
B^{\prime} S\left(S^{\prime} S\right)^{-1} S^{\prime} \mathbf{v}=\mathbf{0},
$$

where $B=\left\{\mathbf{b}_{i}^{\prime}\right\}$ and $S=\left\{\mathbf{S}_{i}^{\prime}\right\}$ are the matrices formed by stacking the vectors $\mathbf{b}_{i}^{\prime}=\left(1, Y_{i} X_{i} \exp \left(-X_{i} \psi_{0}\right)\right)$ and $\mathbf{S}_{i}^{\prime}$, respectively, and $\mathbf{v}=\left\{v_{i}\right\}$ is a column vector with elements given by $v_{i}=Y_{i} \exp \left(-X_{i} \psi_{0}\right)-\alpha_{0}$. It is thus apparent that the GMM estimator combines the instruments in the projection $S\left(S^{\prime} S\right)^{-1} S^{\prime} B$, that is, the multiple instruments for each individual are replaced by the linear projection of $\mathbf{b}_{i}$ onto the space spanned by $S$; alternatively put, the combined instrumental variable can be thought of as the prediction from a linear regression of $\mathbf{b}_{i}$ on the instruments $\mathbf{S}_{i}$.

For the binary variables case considered here, we have that

$$
Y_{i} X_{i} \exp \left(-X_{i} \psi_{0}\right)=Y_{i} X_{i} \exp \left(-\psi_{0}\right),
$$


so that the one-step GMM can be thought of combing the instruments simply using the linear projection of $Y X$ onto the space spanned by $\mathbf{S}$. The one-step GMM estimator for the double-logistic SMM estimator also has the form of a linear projection of $\mathbf{b}_{i}$ onto the space spanned by $S$, but here $\mathbf{b}_{i}^{\prime}=\left(1, q_{i}\left(\psi_{0} ; \widehat{\boldsymbol{\beta}}\right)\{1-\right.$ $\left.\left.q_{i}\left(\psi_{0} ; \widehat{\boldsymbol{\beta}}\right)\right\} X_{i}\right)$. For both the multiplicative and logistic SMMs, these are the simplified combinations of multiple instruments of Bowden and Vansteelandt (2011).

In the simple setup involving only binary variables, the one-step GMM estimator for the multiplicative SMM can be expressed as a linear 2SLS estimator. Following Angrist (2001), note that $\exp \left(-\psi_{0} X\right)=(1-$ $X)+X \exp \left(-\psi_{0}\right)$ and, therefore,

$$
Y \exp \left(-\psi_{0} X\right)-\alpha_{0}=Y(1-X)+Y X \exp \left(-\psi_{0}\right)-\alpha_{0} .
$$

Hence, the moment conditions can be expressed as the linear $\left[\operatorname{in} \exp \left(-\psi_{0}\right)\right]$ moments

$$
E\left[\left\{Y(1-X)+Y X \exp \left(-\psi_{0}\right)-\alpha_{0}\right\} \mathbf{S}\right]=\mathbf{0},
$$

from which we see that the one-step GMM estimator for $\exp \left(-\psi_{0}\right)$ using moment condition (17) is identical to the 2SLS estimator from regressing $Y(X-1)$ on $\widehat{Y X}$, where $\widehat{Y X}$ are the predictions from the linear regression of $Y X$ on $S$.

Multiplying (24) by the risk ratio $\exp \left(\psi_{0}\right)$, we obtain

$$
E\left[\left\{Y X+Y(1-X) \exp \left(\psi_{0}\right)-\gamma_{0}\right\} \mathbf{S}\right]=\mathbf{0},
$$

where $\gamma_{0}=\alpha_{0} \exp \left(\psi_{0}\right)$. In this case, the same estimator as the one-step GMM estimator for $\exp \left(\psi_{0}\right)$ is obtained from a linear instrumental variable estimator where $(X-1) Y$ is instrumented by $\widehat{Y X}$. We will use this result later in Section 6 when deriving results for local risk ratios.

We now move on to the optimal combination of instruments. As we discussed at the end of Section 4.2, Chamberlain (1987) established efficiency results for GMM estimators. We describe these results in terms of a simple multiplicative SMM and its three moment conditions

$$
E\left\{Y \exp \left(-X \psi_{0}\right)-\alpha_{0} \mid Z=z\right\}=0,
$$

for $z=0,1,2$. As shown previously, the instruments can be represented by the vector of orthogonal binary instruments $\mathbf{S}$ and the generalized residual

$$
v\left(Y, X ; \delta_{0}\right)=Y \exp \left(-X \psi_{0}\right)-\alpha_{0},
$$

where $\delta_{0}=\left(\alpha_{0}, \psi_{0}\right)^{\prime}$. Using the notation of Newey (1993), the efficient instrument is

$$
d_{\mathrm{opt}}(\mathbf{S})=Q e(\mathbf{S}) / \sigma^{2}(\mathbf{S}),
$$

where $Q$ is any nonsingular matrix,

$$
\begin{aligned}
e(\mathbf{S}) & =E\left\{\frac{\partial v\left(Y, X ; \delta_{0}\right)}{\partial \delta} \mid \mathbf{S}\right\} \\
& =-\left(E\left\{Y \exp \left(-X \psi_{0}\right) X \mid \mathbf{S}\right\}\right), \\
\sigma^{2}(\mathbf{S}) & =E\left\{v^{2}\left(Y, X ; \delta_{0}\right) \mid \mathbf{S}\right\} \\
& =E\left[\left\{Y \exp \left(-X \psi_{0}\right)-\alpha_{0}\right\}^{2} \mid \mathbf{S}\right],
\end{aligned}
$$

which leads to a GMM estimator with asymptotic covariance

$$
\Lambda=\left[E\left\{e(\mathbf{S}) e(\mathbf{S})^{\prime} / \sigma^{2}(\mathbf{S})\right\}\right]^{-1} .
$$

Chamberlain (1987) showed that, when $\mathbf{S}$ comprises multinomially distributed multiple orthogonal binary instruments such that $\mathbf{S S}^{\prime}=\operatorname{diag}(\mathbf{S})$, the asymptotic covariance of the two-step GMM $\left(C_{0}^{\prime} \Omega_{0}^{-1} C_{0}\right)^{-1}=\Lambda$. Hence, we can derive the optimum combination of instrumental variables from the first-order condition for the two-step GMM estimator:

$$
\begin{gathered}
\left\{\sum_{i=1}^{n} \frac{\partial \mathbf{g}_{i}^{\prime}(\boldsymbol{\delta})}{\partial \boldsymbol{\delta}}\right\} W_{n}^{-1}\left(\widehat{\delta}_{1}\right)\left\{\sum_{i=1}^{n} \mathbf{g}_{i}(\boldsymbol{\delta})\right\} \\
=\left\{\sum_{i=1}^{n}\left(\begin{array}{c}
1 \\
Y_{i} \exp \left(-X_{i} \psi_{0}\right) X_{i}
\end{array}\right) \mathbf{S}_{i}^{\prime}\right\} \\
\cdot W_{n}^{-1}\left(\widehat{\delta}_{1}\right)\left\{\sum_{i=1}^{n} \mathbf{g}_{i}(\boldsymbol{\delta})\right\}=\mathbf{0},
\end{gathered}
$$

where $\mathbf{g}_{i}(\boldsymbol{\delta})=\left\{Y_{i} \exp \left(-X_{i} \psi_{0}\right)-\alpha_{0}\right\} \mathbf{S}_{i}, \mathbf{S}_{i}=\left(Z_{i o}\right.$, $\left.Z_{i 1}, Z_{i 2}\right)^{\prime}$ with $Z_{i j}=I\left(Z_{i}=j\right)$, and

$$
\begin{aligned}
& W_{n}\left(\widehat{\delta}_{1}\right) \\
& =\sum_{i=1}^{n}\left(Y_{i} \exp \left(-X_{i} \widehat{\psi}_{1}\right)-\widehat{\alpha}_{1}\right)^{2} \mathbf{S}_{i} \mathbf{S}_{i}^{\prime} \\
& =\left[\begin{array}{cc}
\sum_{i} Z_{i 0} v^{2}\left(Y_{i}, X_{i} ; \widehat{\delta}_{1}\right) & 0 \\
0 & \sum_{i} Z_{i 1} v^{2}\left(Y_{i}, X_{i} ; \widehat{\delta}_{1}\right) \\
0 & 0 \\
0 & 0 \\
& \sum_{i} Z_{i 2} v^{2}\left(Y_{i}, X_{i} ; \widehat{\delta}_{1}\right)
\end{array}\right] .
\end{aligned}
$$

As before, let the matrices $S$ and $B$ be defined as $B=\left\{\mathbf{b}_{i}^{\prime}\right\}$ and $S=\left\{\mathbf{S}_{i}^{\prime}\right\}$, obtained by stacking the vectors $\mathbf{b}_{i}^{\prime}=\left(1, Y_{i} \exp \left(-X_{i} \psi_{0}\right) X_{i}\right)$ and $\mathbf{S}_{i}^{\prime}$, respectively, 
then the way the two-step GMM estimator combines the multiple instruments is given by

$$
\begin{aligned}
& S W_{n}^{-1}\left(\widehat{\delta}_{1}\right) S^{\prime} B \\
& \quad=S \operatorname{diag}\left(\frac{1}{n_{z}} \sum_{i, Z_{i}=z} v^{2}\left(Y_{i}, X_{i} ; \widehat{\delta}_{1}\right)\right)^{-1}\left(S^{\prime} S\right)^{-1} S^{\prime} B,
\end{aligned}
$$

which is a consistent estimate for the optimal instruments. Chamberlain (1987) further showed that $\Lambda$ is also the lower bound for the asymptotic variance of any consistent asymptotically normally distributed estimator of a semiparametric model where the only substantive restriction imposed on the distribution of the data is (26).

\section{MONTE CARLO STUDIES}

\subsection{Multiplicative SMM}

We now present two Monte Carlo simulation studies to demonstrate the properties of GMM estimators with multiple orthogonal binary instruments in models without covariates. First, we consider the multiplicative SMM by generating data from population model $M_{1}$, which satisfies the multiplicative SMM under both the NEM and CMI restrictions. Population model $M_{1}$ is defined so that

$$
\begin{aligned}
E\left(Y \mid X, Z_{1}, Z_{2}\right)=\exp & \left\{\beta_{0}+\left(\beta_{1}+\psi_{0}\right) X+\beta_{2} Z_{1}\right. \\
+ & \left.\beta_{3} Z_{2}+\beta_{4} X Z_{1}+\beta_{5} X Z_{2}\right\},
\end{aligned}
$$

where $\psi_{0}=0.6$ is the treatment effect. To define the distribution of the observed data, we further define $Z$ to follow the marginal distribution given by $P(Z=1)=0.3$ and $P(Z=2)=0.2$, and $P(X=$ $1 \mid Z=z)=p_{10}+0.15 \times z$ for $z=0,1,2$. To define the joint distribution of the observed and potential outcomes, we set the expected treatment-free outcome in the population to be $\alpha_{0}=E\left(Y_{0}\right)=0.19$, which leads to $\alpha_{0}^{*}=\log E\left(Y_{0}\right)=-1.6607$ in moment conditions (18) and (19), and $E(Y)=0.25, \beta_{1}=0.15$, $\beta_{4}=0.6$ and $\beta_{5}=-0.6$. The other parameter values are then numerically found in order for CMI and NEM to hold: $\beta_{0}=-1.6976, \beta_{2}=-0.3186, \beta_{3}=0.2511$ and $p_{10}=0.2321$.

Table 1 presents some estimation results for 10,000 samples of size 10,000 drawn from population model $M_{1}$. Three different versions of the GMM estimator are applied: the first column of Table 1 contains the results of the just-identified model using the multivalued instrument $Z \in\{0,1,2\}$ as a single instrument so

\begin{tabular}{|c|c|c|c|}
\hline \multirow{3}{*}{$\frac{\text { Instruments } S}{\text { Moment conditions }}$} & \multirow{2}{*}{$\frac{\text { Single instrument }}{1, Z}$} & \multicolumn{2}{|c|}{ Multiple instruments } \\
\hline & & 1,2 & $1, Z_{2}$ \\
\hline & (18) or (19) & (18) & (19) \\
\hline \multicolumn{4}{|l|}{ One-step GMM } \\
\hline$\alpha_{0}^{*}$ & $\begin{array}{r}-1.6614 \\
(0.0839) \\
{[0.0843]}\end{array}$ & $\begin{array}{r}-1.6628 \\
(0.0561) \\
{[0.0566]}\end{array}$ & $\begin{array}{r}-1.6599 \\
(0.0561) \\
{[0.0565]}\end{array}$ \\
\hline$\psi_{0}$ & $\begin{array}{c}0.6151 \\
(0.2175) \\
{[0.2168]}\end{array}$ & $\begin{array}{c}0.6102 \\
(0.1358) \\
{[0.1361]}\end{array}$ & $\begin{array}{c}0.6033 \\
(0.1353) \\
{[0.1356]}\end{array}$ \\
\hline \multicolumn{4}{|l|}{ Two-step GMM } \\
\hline$\alpha_{0}^{*}$ & & $\begin{array}{r}-1.6629 \\
(0.0561) \\
{[0.0565]}\end{array}$ & $\begin{array}{r}-1.6598 \\
(0.0561) \\
{[0.0565]}\end{array}$ \\
\hline$\psi_{0}$ & & $\begin{array}{c}0.6095 \\
(0.1355) \\
{[0.1359]}\end{array}$ & $\begin{array}{c}0.6024 \\
(0.1350) \\
{[0.1353]}\end{array}$ \\
\hline Hansen $J$ & & 0.9806 & 0.9793 \\
\hline Rej. freq. $5 \%$ & & 0.0478 & 0.0475 \\
\hline
\end{tabular}
that $\mathbf{S}=(1, Z)^{\prime}$; in the second and third columns, we
TABLE 1

Monte Carlo estimation results for multiplicative SMM

Notes: Sample size 10,000; means based on 10,000 Monte Carlo replications; std. error in brackets; means of estimated standard errors in square brackets; data drawn from population model $M_{1}$ as described in Section 6.1; $\alpha_{0}^{*}=-1.6607$ and $\psi_{0}=0.6$.

present the one- and two-step GMM estimates for moment conditions (18) and (19), respectively, using multiple instruments so that $\mathbf{S}=\left(1, Z_{1}, Z_{2}\right)^{\prime}$.

All of the estimators display a small positive bias for $\psi_{0}=0.6$, and the mean estimated standard errors are very close to the true standard errors. Among the two estimators using multiple instruments, this bias is slightly larger for the estimator based on moment condition (18). There is here a negligible gain in precision from using the two-step GMM estimator as compared to the one-step estimator. However, there is a substantial gain in efficiency from using two instrumental variables rather than one, with the standard error decreasing from 0.22 for the just-identified model to 0.14 for the two-step GMM estimators. This is because the GMM projection (23) in this case is not linear in $Z$, even though the conditional probabilities $P(X=1 \mid Z)$ are. More specifically, the coefficient on $Z_{2}$ in the regression of $Y X$ on $\left(1, Z_{1}, Z_{2}\right)$ from (23) is actually smaller than that of $Z_{1}$. Under this particular population model (but not generally) the relationship between the coefficients is roughly linear: the average coefficient on $Z_{1}$ is equal to 0.1067 and for $Z_{2}$ it equals 0.0557 . Hence, a single instrument that takes 
the value 1 if $Z=2$ and 2 if $Z=1$ leads to a justidentified estimator which is likely to be almost as efficient as the over-identified GMM estimators. Further simulations show that this is indeed the case, with the just-identified estimator for $\psi_{0}$ just described having an average of 0.6077 and a standard error of 0.1375 , which are both virtually identical to those of the overidentified GMM estimators.

We repeated the analysis above for a similar design to $M_{1}$ but with the instrument $Z$ taking the six values $0,1, \ldots, 5$; full details of this design are available from the authors. The GMM estimators are again well behaved. Using moment conditions (19), the mean based on 10,000 Monte Carlo estimates using the twostep GMM estimator is 0.5966 with a standard error 0.0801 ; the mean estimated standard error equals 0.0806 . The rejection frequency of the $J$-test is $5.1 \%$ at the $5 \%$ level.

Returning to the design with $Z$ taking the values $0,1,2$, we modify population model $M_{1}$ so as to study how the multiplicative GMM performs when $Z$ does not satisfy the key conditions of an instrumental variable. We do this by keeping all $M_{1}$ parameters the same but making the "instrument" $Z_{1}$ invalid. This is done by specifying

$$
\begin{aligned}
& E\left(Y \mid X, Z_{1}, Z_{2}\right) \\
& =\exp \left\{\beta_{0}+\left(\beta_{1}+\psi_{0}\right) X+\left(\beta_{2}+\phi\right) Z_{1}\right. \\
& \left.+\beta_{3} Z_{2}+\beta_{4} X Z_{1}+\beta_{5} X Z_{2}\right\},
\end{aligned}
$$

with $\phi=0.15$. In this case, the CMI assumption is violated as $E\left[Y_{0} \mid Z=0\right]=E\left[Y_{0} \mid Z=2\right]=0.19$ as before, but now $E\left[Y_{0} \mid Z=1\right]=0.2207$. The GMM estimators are now severely biased upwards. The mean based on 10,000 Monte Carlo estimates of the twostep GMM estimator using moments (19) is equal to 1.1191 , with a standard error of 0.1681 . The mean (variance) of Hansen's $J$-test is equal to 3.56 (3.70) with a rejection frequency at the $5 \%$ level of $34 \%$. If instead we change the coefficient on $Z_{2}$ to $\beta_{3}+0.15$, we get a much smaller bias, with the mean (std. error) of the estimator equal to 0.6452 (0.1370), but the rejection frequency of the $J$-test is now much larger, namely, $93 \%$ at the $5 \%$ level. This difference is due to the fact that, as highlighted above, in this case $Z_{1}$ is a stronger instrument than $Z_{2}$, in the sense the SMM estimator is more precise using $Z_{1}$ than when using $Z_{2}$ as an instrument. For example, in the original design where both instruments are valid, using only $Z_{1}$ as an instrument resulted in the median of the 10,000 estimates to be equal to 0.6009 with the interquartile range equal to 0.1967 , whereas using only $Z_{1}$ as an instrument resulted in a median of 0.6242 , with a much larger interquartile range of 1.5253 . If the bias is due to a violation of the CMI assumption for $Z_{1}$, the estimator based on $Z_{2}$ does not have enough precision to reject the null that both moment conditions are valid as frequently as for when $Z_{2}$ is invalid, as the estimator based on $Z_{1}$ is more precise and the test has more power.

\subsection{Logistic SMM}

To investigate the performance of the GMM estimators for the logistic SMM, we generate data from population $M_{2}$ satisfying the logistic SMM model and its corresponding NEM and CMI identification restrictions. More specifically, the data are generated from

$$
\begin{aligned}
E\left(Y \mid X, Z_{1}, Z_{2}\right)=\operatorname{expit}\left\{\beta_{0}+\left(\beta_{1}+\psi_{0}\right) X+\beta_{2} Z_{1}\right. \\
\left.+\beta_{3} Z_{2}+\beta_{4} X Z_{1}+\beta_{5} X Z_{2}\right\},
\end{aligned}
$$

where the treatment effect is again $\psi_{0}=0.6$. Similarly to model $M_{1}$, we set $P(Z=1)=0.3, P(Z=2)=0.2$, $P(X=1 \mid Z=z)=p_{10}+0.15 \times z, E\left(Y_{0}\right)=0.19$, $E(Y)=0.25, \beta_{1}=0.15, \beta_{4}=-0.6$ and $\beta_{5}=0.6$. The other parameters are such that CMI and NEM hold: $\beta_{0}=-1.518, \beta_{2}=0.3183, \beta_{3}=-0.5202$, and $p_{10}=0.4404$.

Table 2 contains estimation results for 10,000 samples of size 10,000 drawn from population model $M_{2}$. Three different versions of the GMM estimator for the logistic SMM are applied: the first column of Table 2 contains the results of the just-identified model using multivalued $Z$ as a single instrument; in the second column, we present the one- and two-step GMM estimates for the 2SGMM using multiple instruments; and the third column contains the corresponding results for the joint-GMM estimator based on (22). Both the 2SGMM and joint-GMM estimators use saturated logistic models for $\boldsymbol{\beta}$ as in (20).

All of the estimators are virtually unbiased and the means of the estimated standard errors are close to Monte Carlo standard errors. There is an efficiency gain from using the instruments separately: the standard error in the just-identified case is 0.1905 , compared to 0.1729 for the $2 \mathrm{SGMM}$ estimator. The performances of the 2SGMM estimator and the GMM estimator using the joint moment conditions are virtually identical. The Hansen $J$-tests are well behaved in both cases. There is no efficiency gain from using the twostep GMM estimators as compared to the one-step estimators in this design. 
TABLE 2

Monte Carlo estimation results for logistic SMM

\begin{tabular}{lccc}
\hline & Single instrument & \multicolumn{2}{c}{ Multiple instruments } \\
\hline Instruments S & $\mathbf{1 , Z}$ & $\mathbf{1}, \boldsymbol{Z}_{\mathbf{1}}, \boldsymbol{Z}_{\mathbf{2}}$ & $\mathbf{1}, \boldsymbol{Z}_{\mathbf{1}}, \boldsymbol{Z}_{\mathbf{2}}$ \\
\hline Moment conditions & Joint/2SGMM & $\mathbf{2 S G M M}$ & Joint-GMM \\
\hline One-step GMM & & & \\
$\alpha_{0}$ & 0.1912 & 0.1905 & 0.1907 \\
& $(0.0168)$ & $(0.0153)$ & $(0.0153)$ \\
$\psi_{0}$ & {$[0.0167]$} & {$[0.0152]$} & {$[0.0152]$} \\
& 0.5970 & 0.6033 & 0.6001 \\
Two-step GMM & $(0.1905)$ & $(0.1729)$ & $(0.1731)$ \\
$\alpha_{0}$ & {$[0.1899]$} & {$[0.1722]$} & {$[0.1721]$} \\
& & & \\
& & 0.1904 & 0.1911 \\
$\psi_{0}$ & & $(0.0153)$ & $(0.0154)$ \\
& & {$[0.0152]$} & {$[0.0152]$} \\
Hansen $J$ & & 0.6038 & 0.5957 \\
Rej. freq. 5\% & & $(0.1729)$ & $(0.1735)$ \\
\hline
\end{tabular}

Notes: Sample size 10,000; means based on 10,000 Monte Carlo replications; std. [error] in brackets; means of estimated standard errors in square brackets; data drawn from population model $M_{2}$ as described in Section $6.2 ; \alpha_{0}=0.19$ and $\psi_{0}=0.6$.

As with the multiplicative SMM, we also find that the estimators behave well for instruments with 6 or even 11 values, although we find that the 2 SGMM estimator has a small upward finite sample bias in the designs we considered. For example, for an instrument with values $0,1,2, \ldots, 10$, we get means (std. error) of the two-step GMM estimates of 0.6323 (0.1073) for 2SGMM and 0.5999 (0.1066) for the joint moments GMM estimator. Details of this design are available from the authors.

Finally, we return to the design with $Z$ taking the values $0,1,2$, and modify population model $M_{2}$ so as to study how these estimators perform when $Z$ is not a valid instrumental variable. We keep all parameters the same but make the "instrument" $Z_{2}$ invalid, by changing the parameter of $Z_{2}$ to $\beta_{3}+\tau$ with $\tau=0.25$. The GMM estimators are now severely biased upwards. The mean of 10,000 Monte Carlo estimates of the twostep GMM estimator using the joint moments (22) is equal to 1.2805 , with a standard error of 0.1511 . However, in this case the mean (variance) of Hansen's $J$-test is equal to 1.26 (3.09), with a rejection frequency at the $5 \%$ level of only $8.5 \%$. In contrast, if we instead change the parameter of $Z_{1}$ to $\beta_{2}+\tau$ with $\tau=0.1$, the estimator has a much smaller bias, with a mean of 0.5527 and standard error of 0.1660 , but the $J$-test has much more power in this case as it rejects $49.4 \%$ of the time at the $5 \%$ level. This is explained by the fact that here $Z_{2}$ is a stronger instrument than $Z_{1}$.

\section{LOCAL AVERAGE TREATMENT EFFECTS}

The parameters of the SMMs we have considered thus far are all identified by the assumption of no effect modification by the instruments (NEM). For the case where we have two instruments $Z_{1}$ and $Z_{2}$, recall that the NEM assumption for the identification of the conditional causal relative risk is that

$$
\frac{E\left(Y \mid X, Z_{1}, Z_{2}\right)}{E\left(Y_{0} \mid X, Z_{1}, Z_{2}\right)}=\exp \left(\psi_{0} X\right),
$$

that is, the instruments $Z_{1}$ and $Z_{2}$ do not modify the causal effect of $X$ on the risk. In this section, we consider how the failure of NEM impacts on GMM estimators for additive and multiplicative SMMs with multiple instruments.

Clarke and Windmeijer (2010) review identification results concerning the additive and multiplicative SMMs in the simple case of a single binary instrument where both $X$ and $Y$ are also binary. If the NEM assumption fails, then a causal effect is identified if the instrument $Z$ has causal effect on treatment $X$ and selection is "monotonic". In this simple case, where $Z$ is randomised treatment assignment and $X$ is the selected treatment, selection is monotonic if

$$
P\left(X_{1}-X_{0} \geq 0\right)=1,
$$

that is, subjects cannot defy their treatment assignments in every potential scenario, so that $\left\{X_{1}=0\right.$, $\left.X_{0}=1\right\}$ has zero probability. Under monotonicity, the additive SMM estimator (13) identifies the "local average treatment effect" (LATE), and the multiplicative SMM identifies the "local risk ratio" (LRR), where

$$
\begin{aligned}
\mathrm{LATE} & =E\left(Y_{1}-Y_{0} \mid X_{1}>X_{0}\right) ; \\
\mathrm{LRR} & =\frac{E\left(Y_{1} \mid X_{1}>X_{0}\right)}{E\left(Y_{0} \mid X_{1}>X_{0}\right)} .
\end{aligned}
$$

LATE is the average treatment effect for the subgroup of subjects who actually and counterfactually accept the treatments to which they have been assigned, that is, $X_{1}=1$ and $X_{0}=0$; for this reason, these subjects are also known as "compliers" and LATE is also known as the "complier average causal effect" (CACE). The logistic SMM does not estimate a local causal effect when NEM fails, but for binary outcomes the local odds ratio can be estimated by taking the ratio of LRR 
estimates obtained by fitting multiplicative SMMs to binary $Y$ and $1-Y$.

If we have two instruments, then these instruments could in principle define two different local causal effects, provided that the two instruments can be combined into a single multivalued instrument. We consider using the single $K$-valued instrument $Z \in$ $\{0,1,2, \ldots, K-1\}$ for binary $X$. In this scenario, monotonic selection does not have the convenient "no defiers" interpretation; instead, selection is monotonic if $z>\widetilde{z}$ implies that $X_{z} \geq X_{\tilde{z}}$ with probability 1 , for any two values $z \neq \tilde{z}$ of the instrument. From this, we can define the analogue of (13) for $z>\widetilde{z}$ as

$$
\beta_{z, \tilde{z}}=\frac{E(Y \mid Z=z)-E(Y \mid Z=\widetilde{z})}{E(X \mid Z=z)-E(X \mid Z=\widetilde{z})},
$$

where $\beta_{z, \tilde{z}}=E\left(Y_{1}-Y_{0} \mid X_{z}>X_{\widetilde{z}}\right) \equiv \mathrm{LATE}_{z, \tilde{z}}$ under monotonicity.

The 2SLS estimator for the additive SMM is obtained as the OLS estimator from the regression of $Y$ on $\widehat{X}$, where $\widehat{X}$ is the prediction from the first-stage regression of $X$ on $\mathbf{S}=\left\{1, Z_{1}, \ldots, Z_{K-1}\right\}^{\prime}$ and $Z_{k}=$ $I(Z=k)$. Let monotonicity hold and the values of $Z$ be ordered such that $E(X \mid Z=k)>E(X \mid Z=k-1)$. Imbens and Angrist (1994) show that the 2SLS estimator is consistent for

$$
\beta_{z}=\sum_{k=1}^{K-1} \mu_{k} \beta_{k, k-1},
$$

where

$$
\begin{aligned}
\mu_{k}= & \{E(X \mid Z=k)-E(X \mid Z=k-1)\} \\
& \cdot \frac{\sum_{l=k}^{K-1}\{E(X \mid Z=l)-E(X)\} \pi_{l}}{\sum_{l=0}^{K-1} E(X \mid Z=l)\{E(X \mid Z=l)-E(X)\} \pi_{l}},
\end{aligned}
$$

and $\pi_{l}=P(Z=l)$ such that $0 \leq \mu_{k} \leq 1$ and $\sum_{l=1}^{K-1} \mu_{k}=1$; see also Angrist and Imbens (1995) and Angrist and Pischke (2009). In other words, when NEM fails but selection is monotonic, the 2SLS estimator is not consistent for $E\left(Y_{1}-Y_{0} \mid X=1\right)$, but for a weighted sum of local average treatment effects.

Alternatively, if we define

$$
\beta_{k, 0}=\frac{E(Y \mid Z=k)-E(Y \mid Z=0)}{E(X \mid Z=k)-E(X \mid Z=0)},
$$

then, following the proof given by Angrist and Imbens (1995), it is easily established that

$$
\beta_{z}=\sum_{k=1}^{K-1} \lambda_{k} \beta_{k, 0},
$$

where

$$
\begin{aligned}
\lambda_{k}= & \{E(X \mid Z=k)-E(X \mid Z=0)\} \\
& \cdot \frac{\{E(X \mid Z=k)-E(X)\} \pi_{k}}{\sum_{l=0}^{K-1} E(X \mid Z=l)\{E(X \mid Z=l)-E(X)\} \pi_{l}},
\end{aligned}
$$

such that $\sum_{l=1}^{K-1} \lambda_{k}=1$. However, in this case, $\beta_{z}$ is only a weighted average of the $\beta_{k, 0}$ (i.e., $0 \leq \lambda_{k} \leq 1$ ) if $E(X \mid Z=1)>E(X)$.

We now extend this result to the multiplicative SMM and give an analogous result for local risk ratios. In Section 4.3 we established that the one-step GMM estimator for $\exp \left(-\psi_{0}\right)$ using moment condition (17) was equivalent to a linear 2 SLS estimator because

$$
\begin{aligned}
& Y \exp \left(-X \psi_{0}\right)-\alpha_{0} \\
& \quad=Y(1-X)+Y X \exp \left(-\psi_{0}\right)-\alpha_{0} .
\end{aligned}
$$

We can therefore straightforwardly generalise the above results of Imbens and Angrist (1994) for the additive SMM to the multiplicative SMM for the inverse local risk ratio. As above, let

$$
\begin{aligned}
& e_{k, k-1}^{-\beta} \\
& \quad=\frac{E\{Y(X-1) \mid Z=k\}-E\{Y(X-1) \mid Z=k-1\}}{E(Y X \mid Z=k)-E(Y X \mid Z=k-1)},
\end{aligned}
$$

where

$$
e_{k, k-1}^{-\beta}=\frac{E\left(Y_{0} \mid X_{k}>X_{k-1}\right)}{E\left(Y_{1} \mid X_{k}>X_{k-1}\right)} \equiv \operatorname{ILRR}_{k, k-1}
$$

is the inverse local risk ratio under monotonicity; see Angrist (2001). We then get equivalent results to the above for the linear SMM, namely, the 2SLS estimator for $\exp \left(-\psi_{0}\right)$ in $(28)$ is a consistent estimator of

$$
e_{z}^{-\beta}=\sum_{k=1}^{K-1} \mu_{k} e_{k, k-1}^{-\beta},
$$

where

$$
\begin{aligned}
\mu_{k}= & \{E(Y X \mid Z=k)-E(Y X \mid Z=k-1)\} \\
& \cdot \frac{\sum_{l=k}^{K-1}\{E(Y X \mid Z=l)-E(Y X)\} \pi_{l}}{\sum_{l=0}^{K-1} E(Y X \mid Z=l)\{E(Y X \mid Z=l)-E(Y X)\} \pi_{l}},
\end{aligned}
$$

and so $e_{z}^{-\beta}$ is a weighted average of inverse local risk ratios if $E(Y X \mid Z=k)>E(Y X \mid Z=k-1)$. As in Angrist and Imbens (1995), the weights $\mu_{k}$ are proportional to $E(Y X \mid Z=k)-E(Y X \mid Z=k-1)$, and hence the stronger the instrument, that is, the bigger the impact of the instrument on the regressor $Y X$ in (28), the more weight (29) receives in the linear combination. The second component of the weighting gives more 
weight to the estimates (29) when the values of $Z$ are closer to the center of the distribution of $Z$ (see Angrist and Imbens, 1995, pages 437).

For the local risk ratio, we use the results from Section 4.3 that the one-step GMM estimator for $\exp \left(\psi_{0}\right)$ can be obtained from a linear IV estimator in the additive SMM with $Y X$ as the "outcome" and $Y(X-1)$ as the "treatment", but with instruments a constant and $E(Y X \mid S)$. Let

$$
\begin{aligned}
& e_{k, k-1}^{\beta} \\
& \quad=\frac{E(Y X \mid Z=k)-E(Y X \mid Z=k-1)}{E\{Y(X-1) \mid Z=k\}-E\{Y(X-1) \mid Z=k-1\}},
\end{aligned}
$$

where $e_{k, k-1}^{\beta}=E\left(Y_{1} \mid X_{k}>X_{k-1}\right) / E\left(Y_{0} \mid X_{k}>\right.$ $\left.X_{k-1}\right) \equiv \mathrm{LRR}_{k, k-1}$ under monotonicity. It follows that the multiplicative SMM estimator is consistent for

$$
e_{z}^{\beta}=\sum_{k=1}^{K-1} \tau_{k} e_{k, k-1}^{\beta},
$$

where

$$
\begin{aligned}
\tau_{k}= & \{E(Y(X-1) \mid Z=k)-E(Y(X-1) \mid Z=k-1)\} \\
& \cdot \frac{\sum_{l=k}^{K-1}\{E(Y X \mid Z=l)-E(Y X)\} \pi_{l}}{\sum_{l=0}^{K-1} E\{Y(X-1) \mid Z=l\}\{E(Y X \mid Z=l)-E(Y X)\} \pi_{l}},
\end{aligned}
$$

and hence $e_{Z}^{\beta}$ is a weighted average of local risk ratios if $E(Y X \mid Z=k)>E(Y X \mid Z=k-1)$ and $E\{Y(X-$ 1) $\mid Z=k\}>E\{Y(X-1) \mid Z=k-1\}$.

As an example, consider an instrument that takes the values $Z=\{0,1,2,3\}$, with $Y$ and $X$ generated from a bivariate normal distribution as

$$
\begin{aligned}
X & =I\left(c_{0}+c_{1} Z_{1}+c_{2} Z_{2}+c_{3} Z_{3}-V>0\right), \\
Y & =I\left(b_{0}+b_{1} X-U>0\right), \\
\left(\begin{array}{l}
U \\
V
\end{array}\right) & \sim N\left(\left(\begin{array}{l}
0 \\
0
\end{array}\right),\left(\begin{array}{ll}
1 & \rho \\
\rho & 1
\end{array}\right)\right),
\end{aligned}
$$

with, as before, $Z_{k}=I(Z=k)$. Setting $\pi_{l}=P(Z=$ $l)=0.25$ for all $l$, the $c_{l}$ parameters are such that $P(X=1 \mid Z=l)=0.1+0.1 \times l, b_{0}=\Phi^{-1}(0.4)$, $b_{1}=0.5$ and $\rho=0.8$. The local risk ratios in this population are $\mathrm{LRR}_{1,0}=1.1585, \mathrm{LRR}_{2,1}=1.3227$ and $\mathrm{LRR}_{3,2}=1.5303$; the population $\tau$-weights are

$$
\tau_{1}=0.3725, \quad \tau_{2}=0.3991, \quad \tau_{3}=0.2285 .
$$

Clarke and Windmeijer (2010) show that the NEM assumption does not hold under this design. However, the instruments are monotonic and so the one-step GMM estimator based on moment conditions (17) identifies the weighted average $\tau_{1} \mathrm{LRR}_{1,0}+\tau_{2} \mathrm{LRR}_{2,1}+$
TABLE 3

Risk ratio estimation results

$\begin{array}{lllllll}e_{1,0}^{\beta} & e_{2,1}^{\beta} & e_{3,2}^{\beta} & e_{z}^{\beta} & \tau_{1} & \tau_{2} & \tau_{3}\end{array}$

$\begin{array}{llllllll}\text { Mean } & 1.1644 & 1.3304 & 1.5415 & 1.3113 & 0.3726 & 0.3995 & 0.2279\end{array}$

$\begin{array}{llllllll}\text { St. dev. } & 0.0946 & 0.1213 & 0.1601 & 0.0377 & 0.0268 & 0.0321 & 0.0216\end{array}$

Notes: Estimation results from 10,000 Monte Carlo replications. Sample size 40,000.

$\tau_{3} \mathrm{LRR}_{3,2}=1.3090$. Table 3 presents some estimation results confirming this, for a sample of size 40,000 and for 10,000 Monte Carlo replications. Using the twostep GMM results, the Hansen $J$-test rejects the null $47 \%$ of the time at the $5 \%$ level, therefore clearly having power to reject this violation of the NEM assumption.

\section{THE EFFECT OF ADIPOSITY ON HYPERTENSION}

\subsection{Binary Exposure}

Timpson et al. (2009) used multiple genetic instruments to estimate the causal effect of adiposity on hypertension from the Copenhagen General Population Study; full details of the variable definitions and selection criteria are given in that paper. We apply the procedures described above to reanalyse these data using additive, multiplicative and logistic SMMs, using the same genetic markers as instruments for adiposity. Furthermore, our sample includes additional individuals who have been recruited into the study since the previous study was published; the total number of individuals in our analyses is 55,523.

The binary outcome variable is an indicator of whether an individual has hypertension, which is defined as a systolic blood pressure of $>140 \mathrm{mmHg}$, diastolic blood pressure of $>90 \mathrm{mmHg}$, or the taking of antihypertensive drugs. The intermediate adiposity phenotype is being overweight, defined as having a BMI $>25$. The two Single Nucleotide Polymorphisms (SNPs) that were used as instruments by Timpson et al. (2009) and that have been consistently shown to relate to BMI and adiposity are the FTO (rs9939609) and MC4R (rs17782313) loci; see Frayling et al. (2007) and Loos et al. (2008). Lawlor et al. (2008) provide further details on the use of genes as instruments in Mendelian randomisation studies.

FTO is specified as having three categories: no risk alleles (homozygous TT), one risk allele (heterozygous AT) and two risk alleles (homozygous AA). Due to the 
TABLE 4

Combinations of instruments

\begin{tabular}{lccc}
\hline FTO & MC4R & $\boldsymbol{Z}$ & Freq. \\
\hline 0 & 0 & 0 & 0.20 \\
0 & 1 & 1 & 0.15 \\
1 & 0 & 1 & 0.27 \\
1 & 1 & 2 & 0.21 \\
2 & 0 & 2 & 0.09 \\
2 & 1 & 3 & 0.07 \\
\hline
\end{tabular}

nature of the association between $M C 4 R$ and adiposity (a dominant genetic model), $M C 4 R$ is specified as having two categories: no risk alleles (TT) versus one or two risk alleles (CT or CC). Combining the two instruments together results in an instrument with 6 different values, but we found that two pairs of combinations of alleles gave the same predicted value of being overweight; this is also true for the projection in the multiplicative SMM. We therefore condensed the number of values of the instrument to four. The combinations for the four values are given in Table 4. Table 5 gives the frequency distributions for the hypertension $(Y)$ and overweight $(X)$ variables.

The estimation results for the linear, multiplicative and logistic SMM estimators are presented in Table 6. The instrument set for the GMM estimators is $\mathbf{S}=$ $\left(1, Z_{1}, Z_{2}, Z_{3}\right)^{\prime}$. For the linear SMM, the 2SLS and two-step GMM estimates are virtually identical to the OLS estimate. As the F-statistic in the regression of overweight on $\mathbf{S}$ is equal to 113 , this is not due to a weak instrument problem. The OLS estimate of the risk difference is quite large and equal to 0.20 (95\% CI $0.19 ; 0.21)$. The two-step GMM estimate is almost the same and equal to 0.21 (95\% CI 0.05-0.37), but clearly the $95 \%$ confidence interval is much wider for the twostep GMM estimate than it is for OLS. The $J$-test does not reject the null of the validity of the model assumptions, including the NEM assumption, and therefore

TABLE 5

Frequency distributions for the hypertension $(Y)$ and overweight $(X)$ variables

\begin{tabular}{|c|c|c|c|c|c|c|c|c|c|c|}
\hline \multicolumn{3}{|c|}{ All } & \multicolumn{2}{|c|}{$Z=\mathbf{0}$} & \multicolumn{2}{|c|}{$Z=1$} & \multicolumn{2}{|c|}{$Z=2$} & \multicolumn{2}{|c|}{$Z=\mathbf{3}$} \\
\hline & \multicolumn{2}{|c|}{$X$} & \multicolumn{2}{|c|}{$X$} & \multicolumn{2}{|c|}{$X$} & \multicolumn{2}{|c|}{$X$} & \multicolumn{2}{|c|}{$X$} \\
\hline$Y$ & 0 & 1 & 0 & 1 & 0 & 1 & 0 & 1 & 0 & 1 \\
\hline 0 & 0.18 & 0.12 & 0.19 & 0.12 & 0.19 & 0.12 & 0.17 & 0.13 & 0.16 & 0.13 \\
\hline 1 & 0.25 & 0.44 & 0.27 & 0.42 & 0.26 & 0.43 & 0.23 & 0.46 & 0.23 & 0.48 \\
\hline
\end{tabular}

these results indicate that there may not be much confounding bias in the OLS results. We find similar results for the multiplicative and logistic SMMs. The GMM estimates are virtually identical to the Gamma and the logistic regression estimates, respectively, and all estimates indicate that being overweight leads to hypertension. The Gamma estimate for the risk ratio is equal to 1.35 (95\% CI, 1.33-1.36), whereas the twostep GMM estimate is equal to 1.36 (95\% CI 1.08 1.72). We present and compare the multiplicative SMM results to that of the Gamma generalised linear model with a log link here, because moment conditions (17)(19) when using $X$ as an instrument for itself are equivalent to the first-order condition of the Gamma with log link GLM. The logistic regression odds ratio is equal to 2.58 (95\% CI, 2.49-2.68) and the two-step GMM estimate is equal to 2.87 (95\% CI 1.25-6.55). All estimation results indicate a large causal effect of adiposity on hypertension.

\subsection{Continuous Exposure}

Following Vansteelandt and Goetghebeur (2003), we can use the same GMM format to estimate the logistic SMM with a continuous exposure $X$. With a continuous exposure, parametric modelling assumptions have to be made in order to identify causal parameters. As in Vansteelandt and Goetghebeur (2003) and Vansteelandt et al. (2011), we impose that the exposure effect is linear in the exposure on the log-odds ratio scale and independent of the instrumental variable:

$$
\frac{\operatorname{odds}(Y=1 \mid X, Z)}{\operatorname{odds}\left(Y_{0}=1 \mid X, Z\right)}=\exp \left(\xi_{0} X\right)
$$

where odds $(Y=1 \mid X, Z)=P(Y=1 \mid X, Z) / P(Y=$ $0 \mid X, Z)$. Further, we specify the association model as

$$
\begin{aligned}
\operatorname{logit}\{P(Y=1 \mid X, Z)\}= & \operatorname{logit}\left\{m_{\beta}\left(X, Z_{1}, Z_{2}, Z_{3}\right)\right\} \\
= & \beta_{0}+\beta_{1} X+\beta_{2} Z_{1}+\beta_{3} Z_{2} \\
& +\beta_{4} Z_{3}+\beta_{5} X Z_{1}+\beta_{6} X Z_{2} \\
& +\beta_{7} X Z_{3},
\end{aligned}
$$

and estimate the parameters using the joint moment conditions as in (22).

For the continuous exposure we use $(B M I-\overline{B M I})$, $10(\ln B M I-\overline{\ln B M I})$ and $10(\ln R E L B M I)$, where ln $B M I$ is the natural logarithm of $B M I$, and $\ln R E L B M I$ are the residuals of the regression of $\ln B M I$ on sex, age, age squared, $\ln$ (height) and an age-sex interaction, as used in Timpson et al. (2009) to represent relative BMI. We subtract the mean from $B M I$ and $\ln B M I$ to 
TABLE 6

SMM estimation results of the effect of being overweight on hypertension

\begin{tabular}{|c|c|c|c|c|}
\hline $\begin{array}{l}\text { Additive } \\
\psi_{0}\end{array}$ & $\begin{array}{c}\text { OLS } \\
0.2009 \\
{[0.1932 ; 0.2087]}\end{array}$ & $\begin{array}{c}2 \text { SLS } \\
0.2091 \\
{[0.0485 ; 0.3697]}\end{array}$ & $\begin{array}{c}\text { GMM2 } \\
0.2095 \\
{[0.0489 ; 0.3701]}\end{array}$ & $\begin{array}{c}J \text {-test } \\
0.2956\end{array}$ \\
\hline $\begin{array}{l}\text { Multiplicative } \\
\exp \left(\psi_{0}\right)\end{array}$ & $\begin{array}{c}\text { Gamma } \\
1.3464 \\
{[1.3300 ; 1.3630]}\end{array}$ & $\begin{array}{c}\text { GMM1 } \\
1.3621 \\
{[1.0784 ; 1.7204]}\end{array}$ & $\begin{array}{c}\text { GMM2 } \\
1.3640 \\
{[1.0798 ; 1.7231]}\end{array}$ & $\begin{array}{r}J \text {-test } \\
0.3071\end{array}$ \\
\hline $\begin{array}{l}\text { Logistic } \\
\exp \left(\psi_{0}\right)\end{array}$ & $\begin{array}{c}\text { Logistic regression } \\
2.5823 \\
{[2.4885 ; 2.6797]}\end{array}$ & $\begin{array}{c}\text { GMM1 } \\
2.8317 \\
{[1.2382 ; 6.4759]}\end{array}$ & $\begin{array}{c}\text { GMM2 } \\
2.8656 \\
{[1.2538 ; 6.5489]}\end{array}$ & $\begin{array}{c}J \text {-test } \\
0.2924\end{array}$ \\
\hline
\end{tabular}

Notes: Sample size 55,523. Gamma regression uses log link; multiplicative SMM uses moments (17); logistic SMM uses joint moments (22); instruments, $S=\left\{1, Z_{1}, Z_{2}, Z_{3}\right\} ; 95 \%$ CIs in brackets; $p$-values are reported for the $J$-test.

ensure that zero exposure is part of the data range. We further multiply the $\ln B M I$ and $\ln R E L B M I$ by a factor 10 so that the estimated odds ratio is for an increase in exposure of approximately $10 \%$.

Table 7 presents the two-step estimation results for three separate models for the three exposure measures. Again, we find a strong positive effect of adiposity on hypertension. The estimate of the odds ratio for a oneunit increase in BMI is equal to 1.12 (95\% CI 1.10; 1.67), whereas the estimates for the odds ratios for a $10 \%$ increase in $\ln B M I$ or $\ln$ RELBMI are $1.35(95 \%$ CI 1.10-1.67) and 1.33 (95\% CI 1.09-1.63), respectively, the latter two therefore virtually identical. Also, for these logistic SMM models with continuous exposures, the $J$-test results do not indicate a problem with the model assumptions.

\section{DISCUSSION}

We have shown how the conditional moment conditions that identify additive, multiplicative and logistic SMMs can be used to derive a standard GMM es-

TABLE 7

Estimation results for double-logistic SMM with continuous exposure

\begin{tabular}{lccc}
\hline Exposure & $B M I$ & $\ln B M I$ & $\ln$ RELBMI \\
$\exp \left(\xi_{0}\right)$ & 1.1187 & 1.3546 & 1.3337 \\
& {$[1.0984 ; 1.6705]$} & {$[1.0984 ; 1.6705]$} & {$[1.0929 ; 1.6276]$} \\
$J$-test & 0.4714 & 0.4828 & 0.5004 \\
\hline
\end{tabular}

Notes: Sample size 55,523. Two-step GMM estimates, using joint moments (22). Instruments, $\mathbf{S}=\left\{1, Z_{1}, Z_{2}, Z_{3}\right\}$. BMI and $\ln B M I$ taken in deviation from the mean. $\ln B M I$ and $\ln R E L B M I$ multiplied by a factor $10.95 \%$ CIs in brackets; $p$-values are reported for the $J$-test. timator of the type widely used in econometrics. The key to this formulation is simply to treat the expected exposure-free potential outcome $E\left(Y_{0}\right)$ as a parameter. For simple SMMs without continuous baseline covariates, these estimators are semiparametrically efficient if the identifying instrumental variables are orthogonal binary variables. In these cases, the estimator combines the instruments optimally in the manner proposed by Bowden and Vansteelandt (2011). Another major advantage is that standard GMM routines are available in statistical software packages. We provide example Stata and R syntax in the Appendix for use by applied researchers. These estimation routines provide correct asymptotic inference, even for the logistic SMM, when the two sets of model parameters are estimated jointly, and a simple test for the validity of the SMM moment conditions. We used Monte Carlo studies to show that the Hansen $J$-test can have power to detect violations of the CMI and NEM assumptions. Moreover, if the NEM assumption fails and selection is monotonic, then we have shown that the one-step GMM estimator for the multiplicative SMM is consistent for a weighted average of the instrument-specific local risk ratios.

A characteristic of all estimating equations for SMMs is that the analyst must specify and estimate auxiliary models further to the SMM. Extending the discussion in Section 2.3 to multiple instrumental variables, the estimating equations for G-estimation depend on $E\left(Z_{j}\right)=\mu_{j}$, which must be replaced in the estimating equation by a consistent estimator $\widehat{\mu}_{j}$. To derive the correct asymptotic distribution, the moment conditions for $\widehat{\mu}_{j}$ must be included in the system of moment conditions. For the multiplicative SMM with multiple instruments discussed in Section 4, the ex- 
tended set of moment conditions is

$$
\left(\begin{array}{c}
E\left(Z_{1}-\mu_{1}\right) \\
E\left(Z_{2}-\mu_{2}\right) \\
E\left\{\left(Z_{1}-\mu_{1}\right) Y \exp \left(-\psi_{0} X\right)\right\} \\
E\left\{\left(Z_{2}-\mu_{2}\right) Y \exp \left(-\psi_{0} X\right)\right\}
\end{array}\right)=\left(\begin{array}{l}
0 \\
0 \\
0 \\
0
\end{array}\right) .
$$

The extended moment conditions can easily be incorporated in the Stata and R GMM estimation routines, and we include in the Appendix code that does this for the additive, multiplicative and logistic SMMs.

There are two relative weaknesses of our approach in applications where covariates $\mathbf{C}$ are required for identification, in other words, where $\mathrm{CMI}$ only holds covariate conditionally such that $E\left(Y_{0} \mid Z, \mathbf{C}\right)=E\left(Y_{0} \mid \mathbf{C}\right)$ but $E\left(Y_{0} \mid Z\right) \neq E\left(Y_{0}\right)$. To discuss these weaknesses, consider a multiplicative SMM which does not depend on C but where covariates are still required for identification. In terms of a GMM estimator, the unconditional moment conditions [equivalent to (17) in Section 4.2] are

$$
E\left[\left\{\frac{Y}{\exp \left(\psi_{0} X\right)}-E\left(Y_{0} \mid \mathbf{C}\right)\right\}\left(\begin{array}{l}
\mathbf{S} \\
\mathbf{C}
\end{array}\right)\right]=\mathbf{0},
$$

which can be seen to depend on the extended instrument $\left(\mathbf{S}^{\prime}, \mathbf{C}^{\prime}\right)^{\prime}$ and $E\left(Y_{0} \mid \mathbf{C}\right)$ as well as the SMM itself.

The first weakness is that the efficiency result for two-step GMM discussed above does not hold if $\mathbf{C}$ includes continuous covariates or if the resulting extended instrument cannot otherwise be represented by a set of the mutually orthogonal binary variables. In such scenarios, the two-step GMM estimator is only locally efficient given the unconditional moments, which here are (17). Newey (1993) discusses different approaches to improve efficiency, for example, using a power-series expansions of the instruments.

The second weakness is that consistency of the GMM estimator now depends on the model for $E\left(Y_{0} \mid \mathbf{C}\right)$ being correctly specified. By definition, this model cannot be empirically tested for misspecification because it is determined by the SMM; but the consequence of misspecifying it is an inconsistent GMM estimator. In contrast, the G-estimators and the doublelogistic SMM estimator discussed in Section 2 require only that $E(Z \mid \mathbf{C})$ is correctly specified, which can be empirically tested for misspecification. Likewise, the doubly robust estimating equations proposed by Tan (2010) depend on covariate-conditional models for $Z$, $X$ given $Z$, and $Y$ given $X$ and $Z$, all of which can be tested for misspecification. The doubly robust property is attractive in theory, but these estimators are not avail- able in standard software, and further work is required to explore fully, rather than locally, efficient choices of weights for the estimating equations. Further work on the GMM estimators proposed here with continuous covariates might investigate the bias and efficiency of GMM estimators, both asympotically and in finite samples, compared to existing estimators for SMMs; see Okui et al. (2012).

\section{APPENDIX: STATA AND R SYNTAX}

In this section we present example Stata (version 11) and R (version 2.13.1) syntax to fit SMMs using generalised method of moments routines. Our example code uses the notation of $Y$ the outcome, $X$ the exposure and two instrumental variables, $Z_{1}, Z_{2}$, in addition to the constant vector of 1 's. Both syntaxes easily generalise to more instruments and allow different association models in the double logistic SMM.

In both Stata and $\mathrm{R}$ it is possible to specify analytic first derivatives, which we find greatly reduces the time for the models to fit. Also, both syntaxes allow the inclusion of covariates. We have not included these extra syntaxes here but they are available on request.

\section{Stata Syntax}

The Stata syntax uses the gmm command; and \{ey0 0 denotes $E\left(Y_{0}\right)$ the mean exposure free potential outcome. After fitting each SMM using two-step estimation we perform the Hansen over-identification test using the estat overid post-estimation command. The gmm command automatically includes a vector of 1's as instruments to allow estimation of the constant $\left[E\left(Y_{0}\right)\right]$ term, hence, we just need to list $z 1$ and $z 2$ in the instruments () option.

Additive SMM. Here \{psi\} denotes the causal effect (which is a risk difference for a binary outcome).

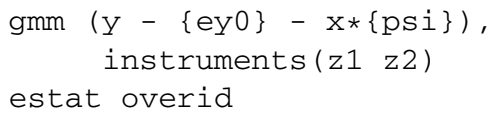

This is equivalent to Stata's built in ivregress command.

ivregress gmm y $(x=z 1 \quad z 2)$

estat overid

Multiplicative SMM. Here \{psi\} denotes the log causal risk ratio, and hence we display the exponentiated estimate using the lincom command with its eform option after fitting the model. 


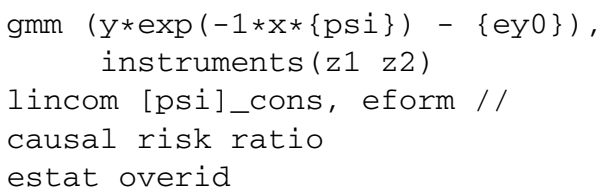

We also give the Stata syntax for the alternative Multiplicative SMM moments. Here $\{$ logey0 $\}$ denotes $\log \left\{E\left(Y_{0}\right)\right\}$ and so we additionally display the exponentiated form of this parameter after fitting the model.

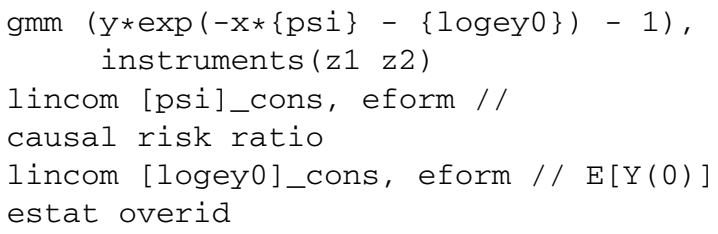

Expanded moments for multiplicative SMM.

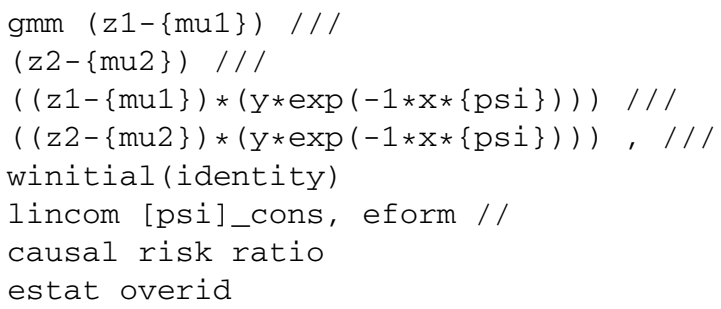

Logistic SMM. Here \{psi\} denotes the log causal odds ratio. In the joint estimation we use the gmm command's linear predictor substitution syntax (we denote the linear predictor for the association model by $\{\mathrm{xb}:\})$. We collect the association and causal model parameter estimates in a matrix called from; we then use these estimates as initial values in the joint estimation. Also, in the joint estimation we specify the winitial (unadjusted, independent) option so that the moments are assumed to be independent in the first step of estimation. Note in Stata, $\operatorname{invlogit}(x)=\operatorname{expit}(x)=e^{x} /\left(1+e^{x}\right)$.

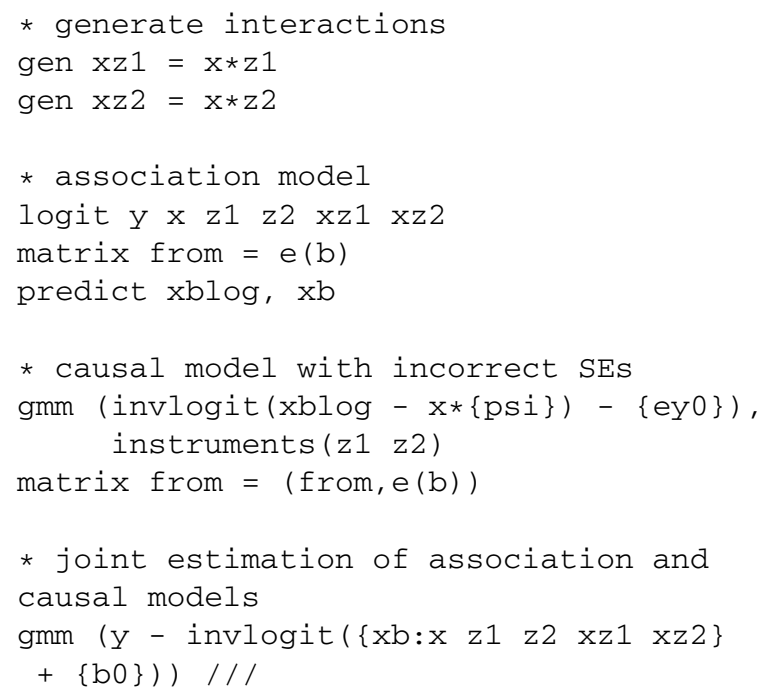

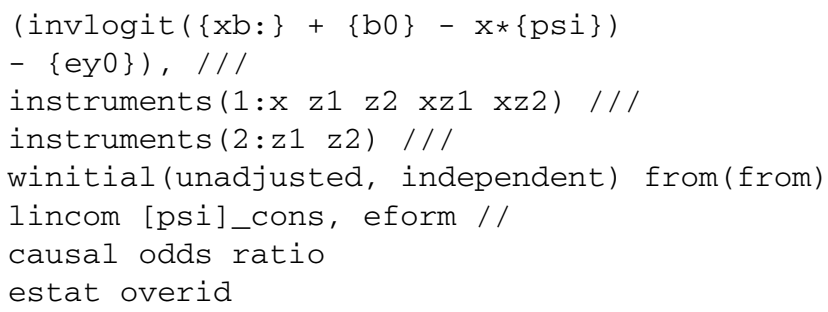

\section{R syntax}

The R syntax uses the gmm ( ) function in the GMM package (Chaussé (2010)), which we first load using library (gmm). After fitting each SMM using two-step estimation we perform the Hansen overidentification test using the specTest () function. The $\mathrm{R}$ code assumes our data is in a matrix called data whose columns contain the values of the variables $Y, X, Z_{1}$ and $Z_{2}$ in this order with column names "Y", "x", "z1", " z2".

In this code we have specified the vcov= " i id" option which assumes the moment conditions are independent. We find specifying this option is necessary for the models to converge on reasonably sized data sets. We also find that changing the optimization algorithm used in the estimation through the method option can reduce the time it takes the models to fit (we find the BFGS and L-BFGS-B methods are the fastest).

Additive SMM. First, we fit the Additive SMM using the gmm ( ) function's formula syntax for linear models.

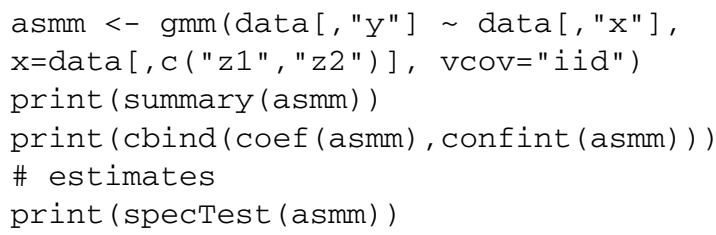

We can also pass the moment conditions to gmm () using its function syntax. In order to do this, we first define a function asmmMoment. ( ) which returns the ASMM moments. This function must have two arguments; the first of which theta denotes the vector of parameters to be estimated, where theta [1] is $E\left(Y_{0}\right)$ and theta [2] is the causal risk difference. The second argument $\mathrm{x}$ is the data matrix; the user must avoid confusion here with the single variable X. In the gmm ( ) function the to option specifies the initial values of the parameter estimates. After we have fitted the model with the call to gmm () we print out the model summary, then the estimates and their 95\% CIs, and finally the over-identification test using specTest (). 


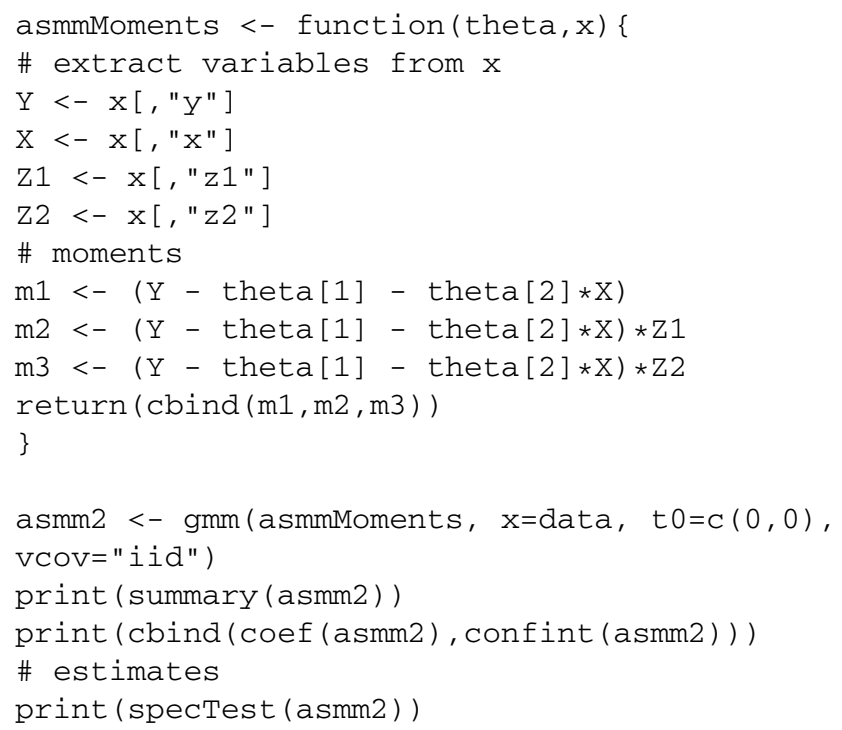

Multiplicative SMM. We again use the gmm ( ) function syntax to fit the Multiplicative SMM. First we define the function msmmMoments () to return the moments. After fitting the model we print the model summary. Here theta [2] is the log causal risk ratio, and so we print the exponentiated form of this parameter.

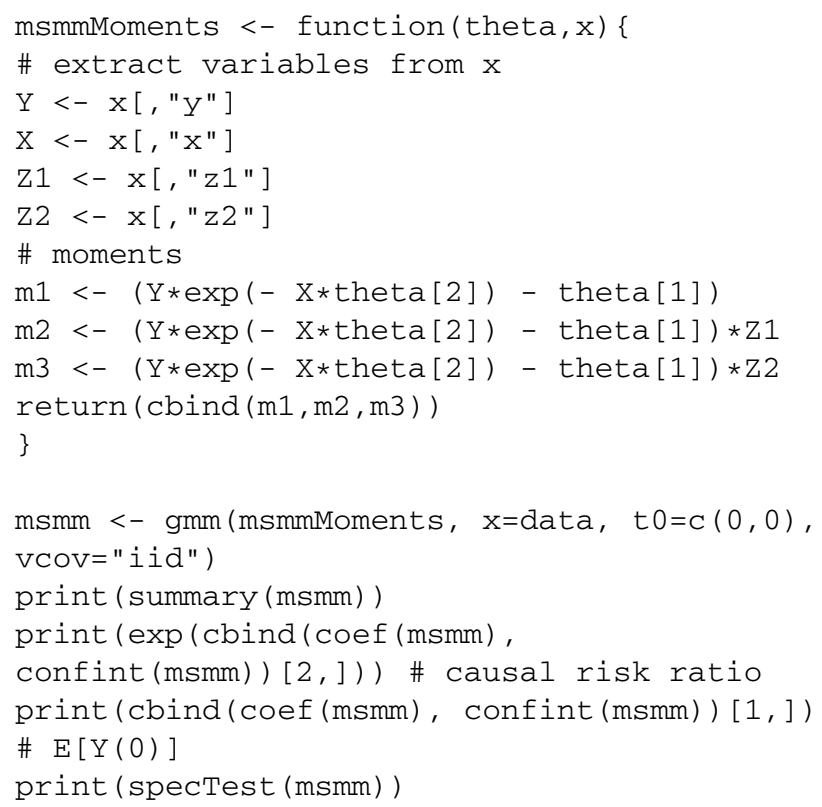

We can also fit the alternative MSMM moments in the same way. Here theta [1] denotes $\log \left\{E\left(Y_{0}\right)\right\}$, and so we print out the exponentiated form of both estimates:

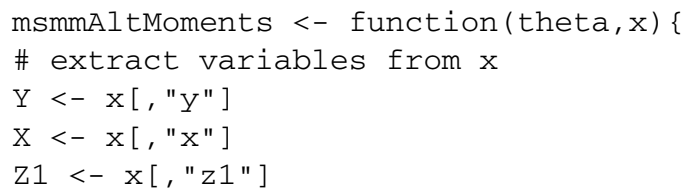

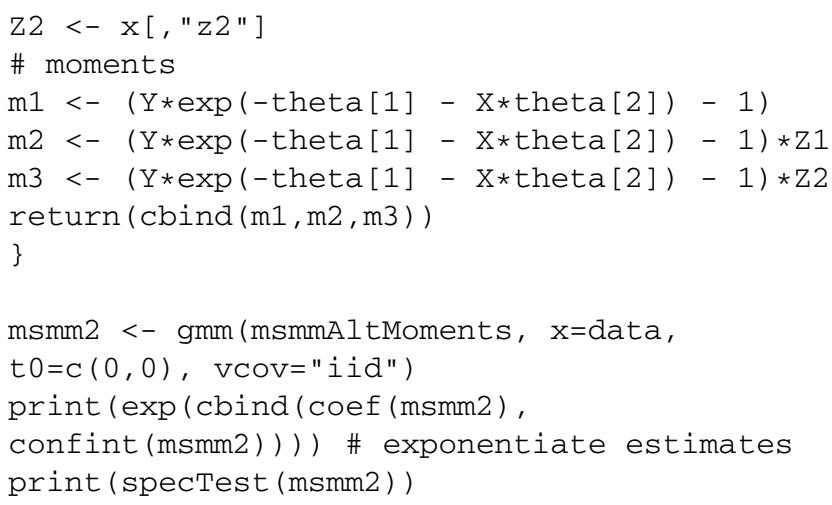

Logistic SMM. In estimation of the logistic SMM, especially with the joint moments, it is important to check that convergence has been reached, either by inspecting the model summary or checking that the model algoInfo\$convergence attribute is equal to 0 . If convergence has not been reached, a higher iteration limit (say, 5000) can be specified in gmm() through the option control=list (maxit= $5000)$. Note in $\mathrm{R} q \operatorname{logis}(p)=\log (p /(1-p))$ and $\operatorname{plogis}(x)=\operatorname{expit}(x)=e^{x} /\left(1+e^{x}\right)$.

First we fit the association model using the $g \operatorname{lm}($ ) function to fit the logistic regression. Again we collect the parameter estimates and predicted values. We then fit the causal model using the function cmMoments () to return its moment conditions. In this function theta [1] denotes $E\left(Y_{0}\right)$ and theta [2] denotes the log causal odds ratio.

In the joint estimation the function 1smmMoments () returns the moment conditions. In this function theta $[1: 6]$ are the coefficients in the association model, theta[7] denotes $E\left(Y_{0}\right)$ and theta [8] denotes the log causal odds ratio.

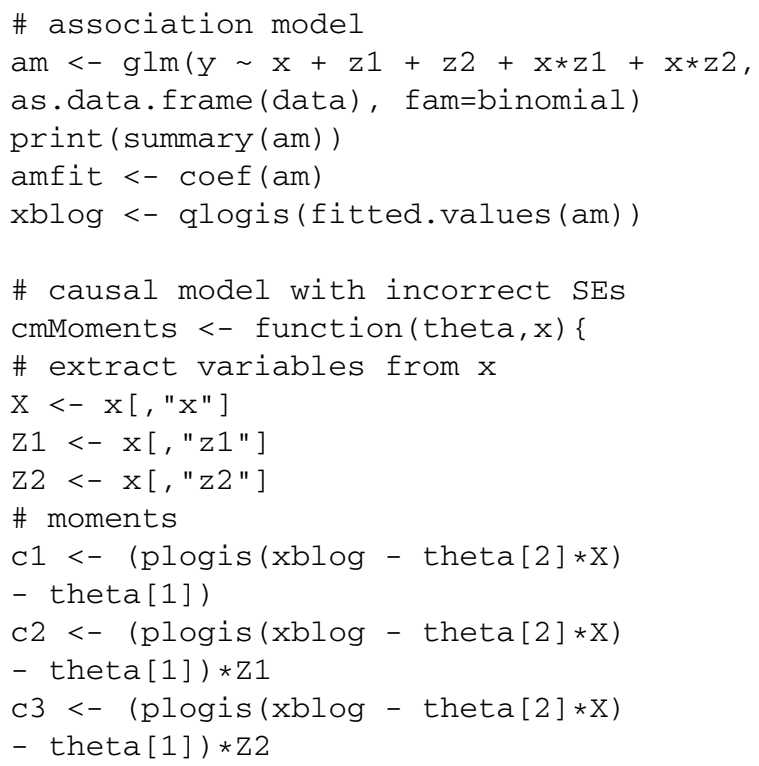




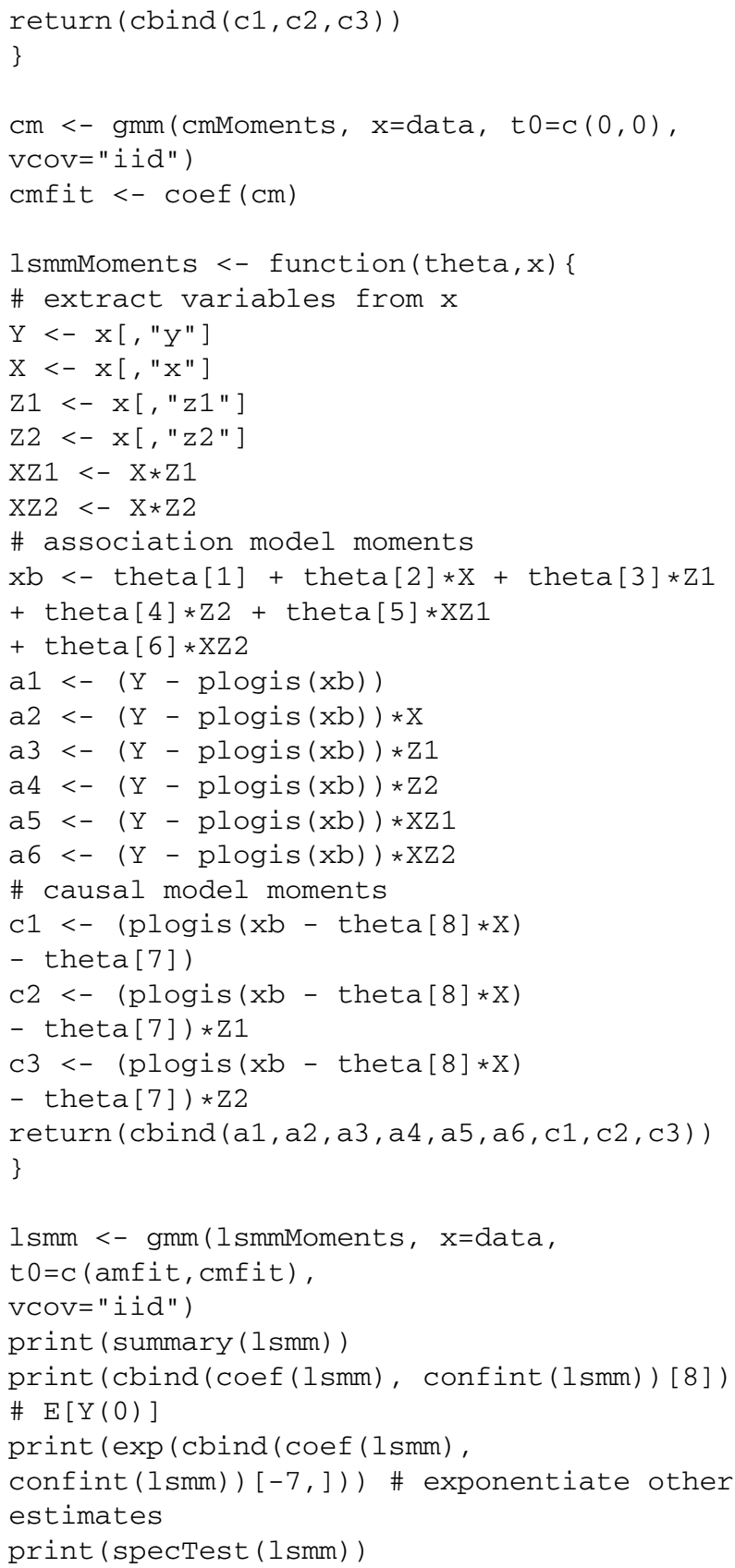

\section{ACKNOWLEDGMENTS}

The authors would like to thank Børge Nordestgaard for access to the Copenhagen General Population Study data. We also thank two anonymous referees and the Editor for very useful comments which improved the manuscript, and George Davey Smith, Nicholas Timpson, Vanessa Didelez, Roger Harbord, Nuala Sheehan and conference participants in London, Lund, Malaga, Manchester and Mannheim for helpful comments.

Research supported in part by UK Economic \& Social Research Council grants RES-060-23-0011 and
RES-576-25-0035, UK Medical Research Council grants G0601625 and G0600705, and European Research Council grant 269874-DEVHEALTH.

\section{REFERENCES}

ANGRIST, J. D. (2001). Estimation of limited dependent variable models with dummy endogenous regressors: Simple strategies for empirical practice. J. Bus. Econom. Statist. 19 2-16. MR1859994

Angrist, J. D. and Imbens, G. W. (1995). Two-stage least squares estimation of average causal effects in models with variable treatment intensity. J. Amer. Statist. Assoc. 90 431-442. MR1340501

Bowden, J. and VANSTEELANDT, S. (2011). Mendelian randomization analysis of case-control data using structural mean models. Stat. Med. 30 678-694. MR2767465

Chamberlain, G. (1987). Asymptotic efficiency in estimation with conditional moment restrictions. J. Econometrics 34305 334. MR0888070

CHAussé, P. (2010). Computing generalized method of moments and generalized empirical likelihood with R. J. Statist. Software 34 1-35.

Clarke, P. S. and WindmeiJer, F. (2010). Identification of causal effects on binary outcomes using structural mean models. Biostatistics 11 756-770.

Davey Smith, G. and Ebrahim, S. (2003). 'Mendelian randomization': Can genetic epidemiology contribute to understanding environmental determinants of disease? International J. Epidemiology 32 1-22.

Frayling, T. M., Timpson, N. J., Weedon, M. N., Zeggini, E., Freathy, R. M., Lindgren, C. M., Perry, J. R. B., Elliott, K. S., LANGo, H., Rayner, N. W., Shields, B., Harries, L. W., BarRett, J. C., Ellard, S., Groves, C. J., Knight, B., Patch, A.-M., Ness, A. R., Ebrahim, S., Lawlor, D. A., Ring, S. M., Ben-Shlomo, Y., JARvelin, M.-R., Sovio, U., Bennett, A. J., Melzer, D., Ferrucci, L., Loos, R. J. F., Barroso, I., Wareham, N. J., Karpe, F., Owen, K. R., CARdon, L. R., Walker, M., HitMAN, G. A., PAlmer, C. N. A., DONey, A. S. F., Morris, A. D., Smith, G. D., Hattersley, A. T. and MCCARTHY, M. I. (2007). A common variant in the FTO gene is associated with body mass index and predisposes to childhood and adult obesity. Science 316 889-894.

Gouriéroux, C., Monfort, A. and Renault, E. (1996). Twostage generalized moment method with applications to regressions with heteroscedasticity of unknown form. J. Statist. Plann. Inference 50 37-63. MR1396448

HANSEN, L. P. (1982). Large sample properties of generalized method of moments estimators. Econometrica 50 1029-1054. MR0666123

HERnÁN, M. A. and RoBIns, J. M. (2006). Instruments for causal inference: An epidemiologist's dream? Epidemiology 17 360372.

Imbens, G. W. and ANGRIst, J. (1994). Identification and estimation of local average treatment effects. Econometrica $\mathbf{6 2}$ 467-476.

KATAN, M. B. (1986). Apolipoprotein E isoforms, serum cholesterol, and cancer. Lancet 327 507-508. 
Lawlor, D. A., Harbord, R. M., Sterne, J. A. C., TimpSON, N. and SMITH, G. D. (2008). Mendelian randomization: Using genes as instruments for making causal inferences in epidemiology. Stat. Med. 27 1133-1163. MR2420151

Loos, R. J. F., Lindgren, C. M., Li, S., Wheeler, E., BARosso, I. et al. (2008). Common variants near MC4R are associated with fat mass, weight and risk of obesity. Nature Genetics 40 768-775.

Mullahy, J. (1997). Instrumental variable estimation of Poisson regression models: Application to models of cigarette smoking behavior. Rev. Econom. Stat. 79 586-593.

NEWEY, W. K. (1993). Efficient estimation of models with conditional moment restrictions. In Econometrics (G. S. Maddala, C. R. Rao and H. D. Vinod, eds.). Handbook of Statist. 11419 454. North-Holland, Amsterdam. MR1247253

Okui, R., Small, D. S., TAN, Z. and Robins, J. M. (2012). Doubly robust instrumental variable regression. Statist. Sinica 22 173-205. MR2933172

Palmer, T. M., Lawlor, D. A., Harbord, R. M., Sheehan, N. A., Tobias, J. H., Timpson, N. J., Smith, G. D. and Sterne, J. A. C. (2012). Using multiple genetic variants as instrumental variables for modifiable risk factors. Stat. Methods Med. Res. 21 223-242. MR2906300

Robins, J. M. (1989). The analysis of randomised and nonrandomised AIDS treatment trials using a new approach to causal inference in longitudinal studies. In Health Service Research Methodology: A Focus on AIDS (L. Sechrest, H. Freeman and A. Mulley, eds.) 113-159. US Public Health Service, National Center for Health Services Research, Washington, DC.

Robins, J. M. (1994). Correcting for non-compliance in randomized trials using structural nested mean models. Comm. Statist. Theory Methods 23 2379-2412. MR1293185

RoBINs, J. M. (2000). Marginal structural models versus structural nested models as tools for causal inference. In Statistical
Models in Epidemiology, the Environment, and Clinical Trials (Minneapolis, MN, 1997) (E. Halloran and D. Berry, eds.). IMA Vol. Math. Appl. 116 95-133. Springer, New York. MR1731682

Robins, J. M., MARK, S. D. and NewEY, W. K. (1992). Estimating exposure effects by modelling the expectation of exposure conditional on confounders. Biometrics 48 479-495. MR1173493

Robins, J. and RotnitzKy, A. (2004). Estimation of treatment effects in randomised trials with non-compliance and a dichotomous outcome using structural mean models. Biometrika 91 763-783. MR2126032

TAN, Z. (2010). Marginal and nested structural models using instrumental variables. J. Amer. Statist. Assoc. 105 157-169. MR2757199

Timpson, N. J., HARbord, R., DAVEy Smith, G., ZACHO, J., TybJÆrg-Hansen, A. and NordestgaArd, B. G. (2009). Does greater adiposity increase blood pressure and hypertension risk? Mendelian randomisation using the FTO/MC4R genotype. Hypertension 54 84-90.

Tsiatis, A. A. (2006). Semiparametric Theory and Missing Data. Springer, New York. MR2233926

Vansteelandt, S. and Goetghebeur, E. (2003). Causal inference with generalized structural mean models. J. R. Stat. Soc. Ser. B Stat. Methodol. 65 817-835. MR2017872

VAnsteelandt, S. and Goetghebeur, E. (2005). Sense and sensitivity when correcting for observed exposures in randomized clinical trials. Stat. Med. 24 191-210. MR2134503

VANSTEELANDT, S., BOWDEN, J., BABANEZHAD, M. and GoetgheBeur, E. (2011). On instrumental variables estimation of causal odds ratios. Statist. Sci. 26 403-422. MR2917963

VAN DER LAAN, M. J., HubBARD, A. and JeWEll, N. P. (2007). Estimation of treatment effects in randomized trials with noncompliance and a dichotomous outcome. J. R. Stat. Soc. Ser. B Stat. Methodol. 69 463-482. MR2323763 